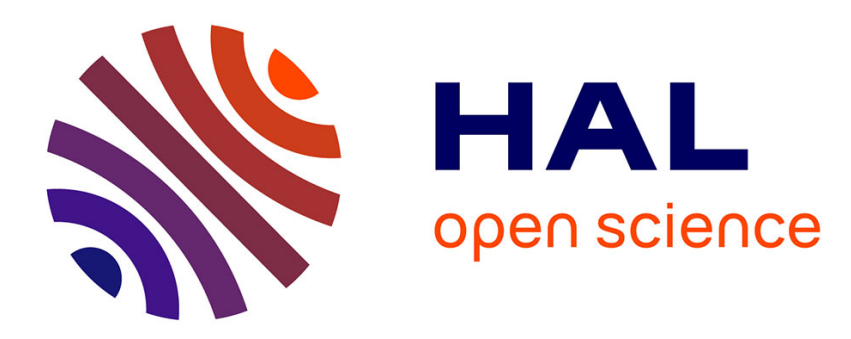

\title{
Solving ordinary differential equations in terms of series with real exponents
}

Dima Grigoriev, Michael F. Singer

\section{To cite this version:}

Dima Grigoriev, Michael F. Singer. Solving ordinary differential equations in terms of series with real exponents. Transactions of the American Mathematical Society, 1991. hal-03053096

\section{HAL Id: hal-03053096 https://hal.science/hal-03053096}

Submitted on 10 Dec 2020

HAL is a multi-disciplinary open access archive for the deposit and dissemination of scientific research documents, whether they are published or not. The documents may come from teaching and research institutions in France or abroad, or from public or private research centers.
L'archive ouverte pluridisciplinaire HAL, est destinée au dépôt et à la diffusion de documents scientifiques de niveau recherche, publiés ou non, émanant des établissements d'enseignement et de recherche français ou étrangers, des laboratoires publics ou privés. 


\title{
SOLVING ORDINARY DIFFERENTIAL EQUATIONS IN TERMS OF SERIES WITH REAL EXPONENTS
}

\author{
D. YU. GRIGOR'EV AND M. F. SINGER
}

\begin{abstract}
We generalize the Newton polygon procedure for algebraic equations to generate solutions of polynomial differential equations of the form $\sum_{i=0}^{\infty} \alpha_{i} x^{\beta_{l}}$ where the $\alpha_{i}$ are complex numbers and the $\beta_{i}$ are real numbers with $\beta_{0}>\beta_{1}>\cdots$. Using the differential version of the Newton polygon process, we show that any such a series solution is finitely determined and show how one can enumerate all such solutions of a given polynomial differential equation. We also show that the question of deciding if a system of polynomial differential equations has such a power series solution is undecidable.
\end{abstract}

When one looks for solutions of differential equations, one is forced to deal with the question: what form can these solutions have? The first natural class of solutions is the set of formal power series. An algorithm to determine whether a system of differential polynomial equations with coefficients in $\mathbb{C}(x)$ has such a solution is given in [DL84]. Even if we only consider algebraic equations, such solutions are not enough; one must consider fractional power series solutions (Puiseux series). In this paper we consider solutions of differential equations of the form $\sum_{i=0}^{\infty} \alpha_{i} x^{\beta_{t}}$ where $\alpha_{i} \in \mathbb{C}$ and $\beta_{i} \in \mathbb{R}$ with $\beta_{0}>\beta_{1}>\cdots$. In $\S 1$, we show (Theorem 1.1) that if such a series satisfies a polynomial differential equation, then the $\beta_{i}$ can have no finite limit point. In particular $\sum_{i=0}^{\infty} x^{1 / i}$ satisfies no polynomial differential equation. This motivates us to introduce the set

$$
\Omega=\left\{\sum_{i=0}^{\infty} \alpha_{i} x^{\beta_{\iota}} \mid \alpha_{i} \in \mathbb{C}, \beta_{i} \in \mathbb{R}, \beta_{0}>\beta_{1}>\cdots \text { and } \lim \beta_{i}=-\infty\right\} .
$$

We will show that $\Omega$ is a field that we call the field of generalized power series. In $\S 2$, we generalize the Newton polygon procedure for algebraic equations to generate solutions of differential equations. In $\S 3$, we show that after a finite number of steps the differential version of the Newton polygon process stabilizes; that is, after generating a finite number of terms of a generalized power series we will reach a point where the remaining terms are uniquely determined. In $\S 4$, we use this to enumerate, for a given differential polynomial

Received by the editors March 15, 1989 and, in revised form, August 24, 1989.

1980 Mathematics Subject Classification (1985 Revision). Primary 12H05; Secondary 13F25.

Key words and phrases. Differential equations, series solutions, Newton polygon.

The authors would like to thank RISC-LINZ for its hospitality and support during the preparation of this paper. The second author was partially supported by NSF Grant DMS-8803109. 
$P\left(x, y, y^{\prime}, \ldots, y^{(n)}\right)$, all generalized power series solutions of $P=0$. We must be careful when we make such a claim since the equation $y^{2}\left(x y^{\prime} / y\right)^{\prime}=0$ has solutions $y=c x^{\alpha}$ for arbitrary $c$ and $\alpha$, showing that such an equation can have an uncountable number of solutions $\left(y^{\prime}=0\right.$ also gives such an example). Our enumeration produces a list $\left\{\varphi_{k}\right\}$ of formulas in the first order language of real closed fields, with each $\varphi_{k}$ having free real variables $\bar{\alpha}_{i k}, \overline{\bar{\alpha}}_{i k}, \beta_{i k}, 0 \leq i \leq n_{k}$. Given $\bar{\alpha}_{i k}, \overline{\bar{\alpha}}_{i k}, \beta_{i k}$ satisfying the formula $\varphi_{k}$, we show that $\sum_{i=0}^{n_{k}}\left(\bar{\alpha}_{i k}+\sqrt{-1} \overline{\bar{\alpha}}_{i k}\right) x^{\beta_{l k}}$ is the initial segment of a uniquely determined generalized power series $y=\sum_{i=0}^{\infty}\left(\bar{\alpha}_{i k}+\sqrt{-1 \bar{\alpha}_{i k}}\right) x^{\beta_{l k}}$, provided that $\lim _{i \rightarrow \infty} \beta_{i k}=-\infty$. If the latter holds, then this generalized power series is a solution of $P=0$. The next terms of the series are determined by a simple recursion formula (cf. Theorem 4.1 of $\S 4$ ). In $\S 5$, we show that the question of deciding if a system of polynomial differential equations has a generalized power series solution is undecidable and discuss some related matters.

\section{THE FIELD OF GENERALIZED POWER SERIES}

Before we turn to generalized power series, we will consider series of the form $\sum_{i=0}^{\infty} \alpha_{i} x^{\beta_{l}}$, where $\alpha_{i} \in \mathbb{C}, \beta_{i} \in \mathbb{R}$, and $\beta_{0}>\beta_{1}>\cdots$ but with no further restriction made on the $\beta_{i}$. Let $\mathbb{C}\left(\left(x^{-1}\right)\right)$ be the field of formal Laurent series in $x^{-1}$. A differential polynomial equation [RI66] with coefficients in $\mathbb{C}\left(\left(x^{-1}\right)\right)$ is an equation of the form $0=F\left(y, y^{\prime}, \ldots, y^{(n)}\right)=\sum_{I} c_{I} y^{\left(k_{0}\right)} \cdots y^{\left(k_{m}\right)}$, where $I=\left(k_{0}, \ldots, k_{m}\right), n \geq k_{0} \geq \cdots \geq k_{m}, c_{I} \in \mathbb{C}\left(\left(x^{-1}\right)\right)$, and distinct $I$ may have different lengths $m+1$. We shall prove the following.

Theorem 1.1. If $y=\sum \alpha_{i} x^{\beta_{\imath}}$ with $\alpha_{i} \in \mathbb{C}, \beta_{i} \in \mathbb{R}, \beta_{0}>\beta_{1}>\cdots$ and $\lim \beta_{i}>$ $-\infty$, then $y$ satisfies no differential polynomial equation with coefficients in $\mathbb{C}\left(\left(x^{-1}\right)\right)$.

We will show, in the corollary to Lemma 1.2 , that substituting $y=\sum \alpha_{i} x^{\beta_{l}}$ into such an equation makes sense (because only a finite number of terms in $y$ contribute to any term of the form $\varepsilon x^{\beta}$ in the expansion of $F\left(y, \ldots, y^{(n)}\right)$ in powers of $x$ ). Our proof of the above theorem relies on the following lemmas and proposition.

Lemma 1.2. Let $\beta_{i} \in \mathbb{R}, i=0,1, \ldots$, where $\beta_{0}>\beta_{1}>\cdots$ and $\lim _{i \rightarrow \infty} \beta_{i}=$ 0 . Then for any integer $m \geq 0$ and $\gamma \in \mathbb{R}, \gamma \neq 0$, there exists an $\varepsilon_{\gamma, m}>0$ such that if

$$
\left|\gamma-\sum_{0 \leq j \leq m} \beta_{i_{j}}\right|<\varepsilon_{\gamma, m}, \quad 0 \leq i_{0} \leq i_{1} \leq \cdots \leq i_{m},
$$

then there exists an $L, 0 \leq L \leq m$, such that $\gamma=\sum_{0 \leq j \leq L} \beta_{i,}$.

Proof. We proceed by induction on $m$. If $m=0$, we can find an $\varepsilon=\varepsilon_{\gamma, 0}>0$ such that in the $\varepsilon$-neighorhood of $\gamma$ there are no elements $\beta_{i}$ except possibly 
$\gamma$ itself. Assume the result is true for $m$. Let $\varepsilon^{\prime}=\frac{|\gamma|}{2(m+2)}$. There exists a $j_{0}$ such that $\beta_{j}<\varepsilon^{\prime}$ for $j>j_{0}$. We now distinguish between two cases.

Case 1. $\gamma \neq \beta_{j}$ for $j=0, \ldots, j_{0}$. In this case we apply the induction hypothesis to $\gamma-\beta_{0}, \ldots, \gamma-\beta_{j_{0}}$ and produce $\varepsilon_{0}, \ldots, \varepsilon_{j_{0}}$, satisfying the conclusion of the lemma with respect to $m$. Let

$$
\varepsilon_{\gamma, m+1}=\min \left\{\varepsilon^{\prime}, \varepsilon_{0}, \ldots, \varepsilon_{j_{0}}\right\} .
$$

If $\left|\gamma-\sum_{0 \leq j \leq m+1} \beta_{i,}\right|<\varepsilon_{\gamma, m+1}$, then $\beta_{i_{0}} \geq \varepsilon^{\prime}$. Indeed if $\beta_{i_{0}}<\varepsilon^{\prime}$, then $\sum_{0 \leq j \leq m+1} \beta_{i,}<|\gamma| / 2$, so $\left|\gamma-\sum_{0 \leq j \leq m+1} \beta_{i_{1}}\right|>|\gamma| / 2 \geq \varepsilon^{\prime} \geq \varepsilon$. Therefore $i_{0} \leq j_{0}$. Since $\left|\gamma-\beta_{i_{0}}-\sum_{1 \leq j \leq m+1} \beta_{i_{1}}\right|<\varepsilon \leq \varepsilon_{i_{0}}$, we have that there exists an $L, 1 \leq L \leq m+1$, such that $\gamma-\beta_{i_{0}}=\sum_{1 \leq j \leq L} \beta_{i_{j}}$. Therefore, $\gamma=\sum_{0 \leq j \leq L} \beta_{i_{j}}$.

Case 2. Assume that $\gamma=\beta_{k}$ for some $0 \leq k \leq j_{0}$. For all $j \neq k, 0 \leq j \leq$ $j_{0}$, apply the induction hypothesis to $\gamma-\beta_{j}$ and produce $\varepsilon_{j}$ satisfying the conclusion of the lemma with respect to $m$. Let

$$
\varepsilon_{\gamma, m+1}=\min \left\{\varepsilon^{\prime}, \varepsilon_{0}, \ldots, \varepsilon_{k+1}, \ldots, \varepsilon_{j_{0}}\right\} .
$$

Assume that $\left|\gamma-\sum_{0 \leq j \leq m+1} \beta_{i}\right|<\varepsilon_{\gamma, m+1}$. If $i_{0}=k$, then we let $L=0$ and the conclusion of the lemma is satisfied. If $i_{0}<j_{0}$ and $i_{0} \neq k$, then we have $\left|\gamma-\beta_{i_{0}}-\sum_{1 \leq j \leq m+1} \beta_{i_{j}}\right|<\varepsilon_{i_{0}}$ and we can argue as before. As in the first case, we show that $i_{0}>j_{0}$ cannot happen.

In our application later of Lemma 1.2 we will assume w.l.o.g. that for any $\gamma \neq 0$, the sequence $\varepsilon_{\gamma, m}$ decreases as $m$ increases.

Corollary. Let $\beta_{i}$ be a decreasing sequence of real numbers. For any $\gamma$ and $m$, there exist only a finite number of solutions $\beta_{i_{0}}, \ldots, \beta_{i_{m}}$ to $\gamma=\beta_{i_{0}}+\cdots+\beta_{i_{m}}$.

Proof. If $\lim \beta_{i}=-\infty$, the result is obvious. Assume that $\lim \beta_{i}=\beta>-\infty$. Replace each $\beta_{i}$ with $\beta_{i}-\beta$ and $\gamma$ with $\gamma-(m+1) \beta$. Note, if $\gamma-(m+1) \beta=0$, we never have $\gamma=\beta_{i_{0}}+\cdots+\beta_{i_{m}}$. We therefore can assume $\lim \beta_{i}=0$ and $\gamma \neq 0$. Let $N$ be an integer such that $\beta_{i}<\varepsilon_{\gamma, m} /(m+1)$ for $i>N$ and $\varepsilon_{\gamma, m}$ as in Lemma 1.2. If $\gamma=\beta_{i_{0}}+\cdots+\beta_{i_{m}}, i_{0} \leq \cdots \leq i_{m}$, and supposing $i_{m}>N$, we shall rewrite this as $\gamma=\sum_{i, \leq N} \beta_{i,}+\sum_{i_{j}>N} \beta_{i,}$. Therefore $\left|\gamma-\sum_{i_{j} \leq N} \beta_{i,}\right|<$ $\varepsilon_{\gamma, m}$, so Lemma 1.2 implies $\gamma=\sum \beta_{i j}$ for some initial subsum of $\sum_{i, \leq N} \beta_{i}$. This contradiction shows that $i_{m} \leq N$ and finishes the proof of the corollary.

This corollary shows that only a finite number of terms of $y=\sum a_{i} x^{\alpha_{t}}$ contribute to any term of the form $c x^{\beta}$ in the expansion of $F\left(y, \ldots, y^{(n)}\right)$ in powers of $x$. 
Lemma 1.3. Let $\beta_{i}$ be as in Lemma 1.2 and assume that $1>\beta_{0}$. The following is true: $\forall j_{0} \exists s_{1} \forall j_{1} \geq s_{1} \exists s_{2} \geq s_{1} \forall j_{2} \geq s_{2} \cdots \exists s_{m} \geq s_{m-1} \forall j_{m} \geq s_{m}$ (if $\beta_{j_{0}}+\cdots+$ $\beta_{j_{m}}=\beta_{j_{0}^{\prime}}+\cdots+\beta_{j_{l}^{\prime}}+q$, where $q \in \mathbb{Z}, j_{0} \leq j_{1} \leq \cdots \leq j_{m}, j_{0}^{\prime} \leq \cdots \leq j_{l}^{\prime}$, $l \leq m$, then $l=m, j_{i}=j_{i}^{\prime}$ for $0 \leq i \leq m$, and $\left.q=0\right)$.

Proof. Note that since $\beta_{i}<1$ for all $i$, we must have $|q| \leq 2 m+1$. For any such $q$ apply the previous lemma in $\beta_{j_{0}}-q$ and produce $\varepsilon_{j_{0}, m}^{(q)}$. (Note that $\beta_{j_{0}}-q \neq 0$.) Let $s_{1}$ be chosen so that $\beta_{s}<\varepsilon_{j_{0}, m}^{(q)} /(m+1)$ for $s \geq s_{1}$. We now proceed by induction on $m$. If $m=0$, then $l=0$ so $\beta_{j_{0}}=\beta_{j_{0}^{\prime}}+q$. Since $\beta_{i}<1, q=0$. Assume $m>0$. By the induction hypothesis, for any $j_{1} \geq s_{1}$ the following is true: $\exists s_{2} \geq s_{1} \forall j_{2} \geq s_{2} \cdots \exists s_{m} \geq s_{m-1} \forall j_{m} \geq s_{m}$ (if $\beta_{j_{1}}+\cdots+\beta_{j_{m}}=\beta_{j_{1}^{\prime \prime}}+\cdots+\beta_{j_{l}^{\prime \prime}}+q$ for $q \in \mathbb{Z}, j_{1} \leq j_{2} \leq \cdots \leq j_{m}$, $j_{1}^{\prime \prime} \leq \cdots \leq j_{l}^{\prime \prime}, l \leq m$, then $l=m$ and $j_{i}=j_{i}^{\prime \prime}$ for $1 \leq i \leq m$ and $\left.q=0\right)$. Letting $\gamma=\beta_{j_{0}}-q$, we have $\left|\gamma-\left(\beta_{j_{0}^{\prime}}+\cdots+\beta_{j_{l}^{\prime}}\right)\right|=\left|\beta_{j_{1}}+\cdots+\beta_{j_{m}}\right|<\varepsilon_{j_{0}, m}^{(q)}$. Therefore Lemma 1.2 implies that $\gamma=\beta_{j_{0}^{\prime}}+\cdots+\beta_{j_{k}^{\prime}}$ for $0 \leq k \leq l$. Note that we are using the remark made after Lemma 1.2 concerning monotonicity of the $\varepsilon$. We therefore have $\beta_{j_{l}}+\cdots+\beta_{j_{m}}=\beta_{j_{k+1}^{\prime}}+\cdots+\beta_{j_{l}^{\prime}}$; so by induction $l-k=m$ (so $k=0$ ) and $j_{1}=j_{1}^{\prime}, \ldots, j_{m}=j_{m}^{\prime}$. Furthermore $\beta_{j_{0}}=\beta_{j_{0}^{\prime}}+q$, so $q=0$.

In the next proposition we need the following definitions. By the degree of the term $c_{I} y^{\left(k_{0}\right)} \cdots \cdots y^{\left(k_{m}\right)}$, where $k_{0} \geq \cdots \geq k_{m}$ in $F$ we mean the number $m+1$. The degree of $F$ is the maximum of the degrees of its terms. Given two terms $t_{1}=c_{I_{1}} y^{\left(k_{0}\right)} \cdots y^{\left(k_{m}\right)}, k_{0} \geq \cdots \geq k_{m}$, and $t_{2}=c_{I_{2}} y^{\left(k_{0}^{\prime}\right)} \cdots y^{\left(k_{j}^{\prime}\right)}, k_{0}^{\prime} \geq \cdots \geq k_{j}^{\prime}$, we say $t_{1}$ is higher than $t_{2}$ if $m>j$ or $m=j$ and $\left(k_{0}, \ldots, k_{m}\right)>\left(k_{0}^{\prime}, \ldots, k_{m}^{\prime}\right)$ in the lexicographical ordering.

Proposition 1.4. If $y=\sum b_{i} x^{\beta_{\imath}}$ with $\beta_{i} \in \mathbb{R}, b_{i} \in \mathbb{C}, 1>\beta_{0}>\beta_{1}>\cdots$ and $\lim \beta_{i}=0$, then $y$ satisfies no differential polynomial equation over $\mathbb{C}\left(\left(x^{-1}\right)\right)$. Proof. Assume not and let $F\left(y, \ldots, y^{(n)}\right)=0$. Let $m$ be the degree of $F$. Lemma 1.3 implies that there are in fact functions $s_{1}\left(j_{0}\right), s_{2}\left(j_{0}, j_{1}\right), \ldots$, $s_{m}\left(j_{0}, \ldots, j_{m-1}\right)$ such that if $j_{i} \geq s_{i}\left(j_{0}, \ldots, j_{i-1}\right)$ for $i=1, \ldots, m$ and $\beta_{j_{0}}+\cdots+\beta_{j_{m}}=\beta_{j_{0}^{\prime}}+\cdots+\beta_{j_{l}^{\prime}}+q$ for $q \in \mathbb{Z}, j_{0} \leq \cdots \leq j_{m}, j_{0}^{\prime} \leq \cdots \leq j_{l}^{\prime}$, $l \leq m$, then $l=m$ and $j_{i}=j_{i}^{\prime}$ for $i=0, \ldots, m$. Denote by $\mathscr{S}$ the set of $(m+1)$-tuples $\left(j_{0}, \ldots, j_{m}\right)$ such that $j_{0} \leq \cdots \leq j_{m}$ and $j_{i} \geq s_{i}\left(j_{0}, \ldots, j_{i-1}\right)$ for $i=1, \ldots, m$. Let $c_{I} y^{\left(k_{0}\right)} \ldots v^{\left(k_{m}\right)}$ be the highest term occurring in $F$ where $I=\left(k_{0}, \ldots, k_{m}\right)$ and let $c_{I}=\sum_{t \leq t_{I}} c_{t}^{(I)} x^{t}$ with $c_{t_{I}}^{(I)} \neq 0$.

Consider $\left(j_{0}, \ldots, j_{m}\right) \in \mathscr{S}$ and let us determine the coefficients of powers of $x$ in $F\left(y, \ldots, y^{(n)}\right)$. Any power of $x$ in this expansion is of the form $x^{\beta_{J_{0}^{\prime}}+\cdots+\beta_{J_{l}^{\prime}}-k_{0}^{\prime}-\cdots-k_{l}^{\prime}+t^{\prime}}$ with $j_{0}^{\prime} \leq \cdots \leq j_{l}^{\prime}, l \leq m$ and some $t^{\prime} \in \mathbb{Z}$ (this comes from a term $\left.c_{I^{\prime}} y^{\left(k_{0}^{\prime}\right)} \cdots y^{\left(k_{l}^{\prime}\right)}\right)$. Lemma 1.3 implies that if

$$
x^{\beta_{J_{0}}+\cdots+\beta_{J_{m}}-k_{0}-\cdots-k_{m}+t}=x^{\beta_{J_{0}^{\prime}}^{\prime}+\cdots+\beta_{J_{l}^{\prime}}-k_{0}^{\prime}-\cdots-k_{l}^{\prime}+t^{\prime}}
$$


then $l=m, \beta_{j_{l}}=\beta_{j_{l}^{\prime}}, i=0, \ldots, m$, and $k_{0}+\cdots+k_{m}-t=k_{0}^{\prime}+\cdots+$ $k_{m}^{\prime}-t^{\prime}$. Therefore the coefficient of $x^{\beta_{J_{0}}+\cdots+\beta_{J_{m}}-k_{0}-\cdots-k_{m}+t_{I}}$ in the expansion of $F\left(y, \ldots, y^{(n)}\right)$ is

$$
\begin{gathered}
\left(b_{j_{0}} \cdots b_{j_{m}}\right) \sum_{k_{0}^{\prime}, \ldots, k_{m}^{\prime}} c_{t^{\prime}}^{\left(I^{\prime}\right)} \sum_{\left(h_{0}, \ldots, h_{m}\right)}\left(\begin{array}{c}
\beta_{h_{0}} \\
k_{0}^{\prime}
\end{array}\right) k_{0}^{\prime} ! \cdots\left(\begin{array}{c}
\beta_{h_{m}} \\
k_{m}^{\prime}
\end{array}\right) k_{m}^{\prime} ! \\
=\left(b_{j_{0}} \cdots b_{j_{m}}\right) P\left(\beta_{j_{0}}, \ldots, \beta_{j_{m}}\right)
\end{gathered}
$$

where the first sum is over all $\left(k_{0}^{\prime}, \ldots, k_{m}^{\prime}\right)$ such that $k_{0}^{\prime}+\cdots+k_{m}^{\prime}-t^{\prime}=$ $k_{0}+\cdots+k_{m}-t_{I}$ and the second sum is over all $\left(h_{0}, \ldots, h_{m}\right)=\left(j_{\sigma(0)}, \ldots, j_{\sigma(m)}\right)$ where $\sigma$ is a permutation of $(0, \ldots, m)$ and

$$
P\left(Z_{0}, \ldots, Z_{m}\right)=\sum_{\left(k_{0}^{\prime}, \ldots, k_{m}^{\prime}\right)} c_{t^{\prime}}^{\left(I^{\prime}\right)} \sum_{\left(i_{0}, \ldots, i_{m}\right)} k_{0}^{\prime} ! \cdots k_{m}^{\prime} !\left(\begin{array}{c}
Z_{i_{0}} \\
k_{0}^{\prime}
\end{array}\right) \cdots\left(\begin{array}{c}
Z_{i_{m}} \\
k_{m}^{\prime}
\end{array}\right)
$$

where the first sum is as before and the second is over all permutations of $(0, \ldots, m)$. Here

$$
\left(\begin{array}{l}
Z_{i} \\
k_{j}
\end{array}\right)=\frac{Z_{i}\left(Z_{i}-1\right) \cdots\left(Z_{i}-k_{j}+1\right)}{k_{j} !} .
$$

Note $P\left(Z_{0}, \ldots, Z_{m}\right) \in \mathbb{C}\left[Z_{0}, \ldots, Z_{m}\right]$. Order the monomials in $Z_{0}, \ldots, Z_{m}$ first by degree and then, for fixed degree, lexicographically. If $\left(i_{0}, \ldots, i_{m}\right)$ is a permutation of $(0, \ldots, m)$, then $Z_{0}^{k_{0}} \cdots Z_{m}^{k_{m}}$ is not less than $Z_{i_{0}}^{k_{0}} \cdots Z_{i_{m}}^{k_{m}}$. Also, for any term $c_{t^{\prime}}^{\left(I^{\prime}\right)} y^{\left(k_{0}^{\prime}\right)} \cdots y_{m}^{\left(k_{m}^{\prime}\right)}$ (where $k_{0}^{\prime} \geq \cdots \geq k_{m}^{\prime}$ ) occurring in $F$ such that $\left(k_{0}^{\prime}, \ldots, k_{m}^{\prime}\right) \neq\left(k_{0}, \ldots, k_{m}\right)$, we have that $Z_{0}^{k_{0}} \cdots Z_{m}^{k_{m}}$ is larger than $Z_{0}^{k_{0}^{\prime}} \ldots Z_{m}^{k_{m}^{\prime}}$, since $\left(k_{0}, \ldots, k_{m}\right)$ was chosen so that $\left(k_{0}, \ldots, k_{m}\right)>\left(k_{0}^{\prime}, \ldots, k_{m}^{\prime}\right)$ in the lexicographic ordering. These two facts imply that the highest term in $P\left(Z_{0}, \ldots, Z_{m}\right)$ is $l c_{t_{t}}^{(I)} Z_{0}^{k_{0}} \ldots Z_{m}^{k_{m}}$, where $l$ is the number of permutations of $(0, \ldots, m)$, leaving $\left(k_{0}, \ldots, k_{m}\right)$ fixed. Therefore, $P$ does not vanish identically. For some $\bar{j}_{0}, P\left(\beta_{\bar{j}_{0}}, Z_{1}, \ldots, Z_{m}\right)$ does not vanish identically. Therefore for some $\bar{j}_{1}>s_{1}\left(\bar{j}_{0}\right), P\left(\beta_{\bar{j}_{0}}, \beta_{\bar{j}_{1}}, Z_{2}, \ldots, Z_{m}\right)$ does not vanish identically. Continuing in this way, we have for some $\bar{j}_{0}, \bar{j}_{1}>s_{1}\left(\bar{j}_{0}\right), \ldots, \bar{j}_{m}>$ $s_{m}\left(\bar{j}_{0}, \ldots, \bar{j}_{m-1}\right)$, that $P\left(\beta_{\bar{j}_{0}}, \ldots, \beta_{\bar{j}_{m}}\right) \neq 0$. This means that the coefficient of $x^{\beta_{j_{0}}+\cdots+\beta_{j_{m}}-k_{0}-\cdots-k_{m}+t_{l}}$ is not zero in the expansion of $F\left(y, \ldots, y^{(n)}\right)$. This contradiction completes the proof of the proposition.

Proof of Theorem 1.1. We assume the theorem is false and derive a contradiction. Let $\lim \alpha_{j}=\alpha>-\infty$ and let $j_{0}$ be an integer such that $\alpha_{j}<\alpha+1$ for $j>j_{0}$. Let $z=\left(y-\sum_{j \leq j_{0}} a_{j} x^{\alpha}\right) x^{-\alpha}$. The term $z$ satisfies a differential polynomial equation with coefficients in the differential field [RI50]

$$
\begin{aligned}
F & =\mathbb{C}\left(\left(x^{-1}\right)\right)\left\langle x^{\alpha_{0}}, \ldots, x^{\alpha_{J_{0}}}, x^{\alpha}\right\rangle \\
& =\mathbb{C}\left(\left(x^{-1}\right)\right)\left(x^{\alpha_{0}}, \ldots, x^{\alpha_{J_{0}}}, x^{\alpha}\right) .
\end{aligned}
$$


Therefore, $F\left(z, z^{\prime}, z^{\prime \prime}, \ldots\right)=F\langle z\rangle$ has finite transcendence degree over $F$. Since $F$ has finite transcendence degree over $\mathbb{C}\left(\left(x^{-1}\right)\right), F\langle z\rangle$ has finite transcendence degree over $\mathbb{C}\left(\left(x^{-1}\right)\right)$. This implies that for some $N>0, z, z^{\prime}, \ldots$, $z^{(N)}$ are algebraically dependent over $\mathbb{C}\left(\left(x^{-1}\right)\right)$. Therefore $z$ satisfies a polynomial differential equation over $\mathbb{C}\left(\left(x^{-1}\right)\right)$. If $z=\sum b_{i} x^{\beta_{\imath}}$, we have $1>\beta_{0}>\cdots$ and $\lim \beta_{i}=0$. Now apply Proposition 1.4 to reach a contradiction and prove the theorem.

Theorem 1.1 implies that if we are looking for series solutions $\sum \alpha_{i} x^{\beta_{l}}$ of polynomial differential equations, we can assume that $\lim \beta_{i}=-\infty$. We therefore make the following definition. A generalized power series is a formal series of the following form: $y=\sum_{i=0}^{\infty} \alpha_{i} x^{\beta_{i}}$ where $\alpha_{i} \in \mathbb{C}, \beta_{i} \in \mathbb{R}, \beta_{0}>\beta_{1}>\cdots$, and $\lim \beta_{i}=-\infty$. (Note that $y$ may consist of a finite number of terms, in which case, there is an $N$ such that $\alpha_{i}=0$ for $i>N$.) We denote by $\Omega$ the set of generalized power series. To prove that $\Omega$ is a field we need the following obvious

Lemma 1.5. If $\beta_{0}>\beta_{1}>\cdots, \beta_{0}^{\prime}>\beta_{1}^{\prime}>\cdots$ and $\lim \beta_{i}=\lim \beta_{i}^{\prime}=-\infty$, then for any $M \in \mathbb{R}$ there are at most a finite number of pairs $(i, j)$ such that $\beta_{i}+\beta_{j}^{\prime}>M$.

Proof. Assume that $\beta_{i}+\beta_{j}^{\prime}>M$ for some $i, j$. We then have $\beta_{0}+\beta_{j}^{\prime}>M$ and so $\beta_{j}^{\prime}>M-\beta_{0}$. This can only be satisfied for a finite number of $j$. Similarly, there are only a finite number of possibilities for $i$. This proves the lemma.

Corollary 1. If $\beta_{0}^{(1)}>\beta_{1}^{(1)}>\cdots, \beta_{0}^{(2)}>\beta_{1}^{(2)}>\cdots, \beta_{0}^{(m)}>\beta_{1}^{(m)}>\cdots$, and $\lim _{i \rightarrow \infty} \beta_{i}^{(j)}=-\infty$ for each $1 \leq j \leq m$, then for any $M \in \mathbb{R}$ there are at most a finite number of $m$-tuples $i_{1}, \ldots, i_{m}$ such that $\beta_{i_{1}}^{(1)}+\cdots+\beta_{i_{m}}^{(m)}>M$.

Proof. Proceed by induction of $m$, taking into account that it is equivalent to the following statement:

$$
\lim _{i_{1} \rightarrow \infty, \ldots, i_{m} \rightarrow \infty}\left(\beta_{i_{1}}^{(1)}+\cdots+\beta_{i_{m}}^{(m)}\right)=-\infty,
$$

and for $m=2$ this was proved in Lemma 1.5.

Corollary 2. $\Omega$ is a differential field.

Proof. Lemma 1.5 implies that the product of two elements of $\Omega$ belongs to $\Omega$. Moreover,

$$
\begin{aligned}
y^{-1} & =\alpha_{0} x^{-\beta_{0}}\left(1+\sum_{i \geq 1} \frac{\alpha_{i}}{\alpha_{0}} x^{\beta_{l}-\beta_{0}}\right)^{-1} \\
& =\alpha_{0}^{-1} x^{-\beta_{0}} \sum_{j \geq 0}(-1)^{j}\left(\sum_{i \geq 1} \frac{\alpha_{i}}{\alpha_{0}} x^{\beta_{l}-\beta_{0}}\right)^{j} .
\end{aligned}
$$


As mentioned above, each $\left(\sum_{i \geq 1} x^{\beta_{\imath}-\beta_{0}}\right)^{j}$ lies in $\Omega$. Furthermore, for any $M,\left(\sum_{i \geq 1} x^{\beta_{l}-\beta_{0}}\right)^{j}$ can contribute to the coefficient of any power $x^{M_{1}}$, where $M_{1} \geq M$ only if $j<\left(M-\beta_{0}\right) /\left(\beta_{1}-\beta_{0}\right)$. Thus the exponents in the expansion of $y^{-1}$ have no finite limit points, proving the corollary.

We shall see that generalized power series appear naturally when one tries to apply a process similar to the classical (i.e. dealing with algebraic functions) Newton polygon process to generating a formal solution of a system of ordinary differential equations. Generalized power series have occurred in other contexts as well. The field $\mathscr{L}$ of series of the form $\sum_{i=0}^{\infty} a_{i} x^{\nu_{l}}$, where $a_{i}, \nu_{i} \in \mathbb{R}$, $\nu_{0}<\nu_{1}<\cdots$, and $\lim \nu_{i}=\infty$, was studied by Levi-Civita [LC54] in the nineteenth century, by Ostrowski [OS35] and Mac Lane [MA39] in the 1930's, and more recently by Laugwitz [LA68], Robinson [RO73], and Lightstone and Robinson [LR75]. Ostrowski studied this field as part of a general investigation of valuation theory. Mac Lane showed that when we let the $a_{i}$ be in $\mathbb{C}$, this field is algebraically closed. The other authors studied $\mathscr{L}$ as a nonarchimedian field (ordered with $x$ as an infinitesimal) with the idea of doing analysis over this field. Clearly $\mathscr{L}$ (when $a_{i} \in \mathbb{C}$ ) and $\Omega$ are isomorphic. We have chosen to work with $\Omega$ because of its relation to asymptotic expansions of a function at $\infty$.

In our generalization of the Newton polygon process, we will need the following technical lemma.

Lemma 1.6. If $y=\sum_{i \geq 1} \alpha_{i} x^{\beta_{\imath}} \in \Omega$ and $P\left(x, Y, \ldots, Y^{(m)}\right)$ is a differential polynomial such that $0 \neq P(y)=\alpha x^{\beta}+$ terms with exponents smaller than $\beta$, $\alpha \neq 0$, then for suitable $N, P\left(\sum_{1 \leq i \leq N} \alpha_{i} x^{\beta_{l}}\right)=\alpha x^{\beta}+$ terms with exponents smaller than $\beta$.

Proof. Consider any term $a_{k} x^{k_{0}} Y^{\left(k_{1}\right)} \ldots Y^{\left(k_{s}\right)}$ of the polynomial $P$, where $a_{k} \in$ $\mathbb{C}$. If some term from the expansion of $x^{k_{0}} y^{\left(k_{1}\right)} \cdots y^{\left(k_{s}\right)}$ makes a contribution to $\alpha x^{\beta}$, then $\beta=k_{0}+\beta_{i_{1}}-k_{1}+\cdots+\beta_{i_{s}}-k_{s}$ for some $i_{1}, \ldots, i_{s}$. Corollary 1 of Lemma 1.5 implies that there are only a finite number of such $i_{1}, \ldots, i_{s}$. The lemma is proved.

\section{DiffERENTIAL VERSION OF THE NEWTON POLYGON PROCESS}

Let the differential polynomial $P \in \mathbb{Q}\left[x, Y, Y^{(1)}, \ldots, Y^{(n)}\right]$ be given. Write $P=P_{0}+\cdots+P_{N}$, where $P_{j}=\sum_{K} \alpha_{K} Y^{\left(k_{1}\right)} \cdots Y^{\left(k_{j}\right)}$ is the homogeneous part of $P$ of degree $j$ with respect to the indeterminants $Y, Y^{(1)}, \ldots, Y^{(m)}, K=$ $\left(k_{1}, \ldots, k_{j}\right)$ is a multi-index with $k_{1} \geq k_{2} \geq \cdots \geq k_{j}$ and $a_{K} \in \mathbb{Q}[x]$. The Newton polygon process that we will now describe produces a generalized power series $y$. If $y \in \Omega$, then this series will furthermore be a solution of $P=0$. Assume that some initial segment

$$
y_{0}=\sum_{1 \leq i \leq s}\left(\bar{\alpha}_{i}+\sqrt{-1} \overline{\bar{\alpha}}_{i}\right) x^{\beta_{l}}
$$


of $y$ has already been produced where $\bar{\alpha}_{i}, \overline{\bar{\alpha}}_{i}, \beta_{i} \in \mathbb{R}$ (we do not exclude the possibility that $\left.y_{0}=0\right)$. Using Taylor's formula, we define $P^{\left(y_{0}\right)}(Y)$ as

$$
P^{\left(y_{0}\right)}(Y)=P\left(y_{0}+Y\right)=\left.\sum_{K} \frac{1}{|K| !} \frac{\partial^{K} P}{\partial Y^{K}}\right|_{Y=y_{0}} Y^{K},
$$

where $K=\left(k_{1}, \ldots, k_{j}\right)$ is a multi-index, $Y^{K}=Y^{\left(k_{1}\right)} \cdots Y^{\left(k_{j}\right)}, \partial^{K} P / \partial Y^{K}=$ $\partial^{j} P / \partial Y^{\left(k_{1}\right)} \cdots \partial Y^{\left(k_{j}\right)}$, and $|K| !=i_{1} ! \cdots i_{l} !$, where $i_{1}$ is the largest $t$ such that $k_{1}=k_{2}=\cdots=k_{t}, i_{2}$ is the largest $t$ such that $k_{i_{1}+1}=k_{i_{1}+2}=\cdots=k_{i_{1}+t}$, etc. We also define $P_{K}^{\left(y_{0}\right)}$ as

$$
\begin{aligned}
P_{K}^{\left(y_{0}\right)} & =\left.\frac{\partial^{K} P}{\partial Y^{K}}\right|_{Y=y_{0}} \\
& \in \mathbb{Q}\left[\bar{\alpha}_{1}, \ldots, \bar{\alpha}_{s}, \overline{\bar{\alpha}}_{1}, \ldots, \overline{\bar{\alpha}}_{s}, \beta_{1}, \ldots, \beta_{s}, \sqrt{-1}\right]\left[x, x^{\beta_{1}}, \ldots, x^{\beta_{s}}\right] .
\end{aligned}
$$

We define the weight $w_{K}$ of the term $P_{K}^{\left(y_{0}\right)} Y^{(K)}$ as $\operatorname{deg}_{x} P_{K}^{\left(y_{0}\right)}-k_{1}-\cdots-k_{j}$. Let $w^{(j)}=\max _{K=\left(k_{1}, \ldots, k_{j}\right)} w_{K}$. We now define the Newton polygon. For every $K$ we mark the point $\left(w_{K}, j\right)$ on $(x, y)$ plane. The convex hull of these points and $(-\infty, 0)$ is denoted by $\mathscr{P}_{y_{0}}$ and is called the Newton polygon of the equation $P=0$ corresponding to the initial segment $y_{0}$. If $n=\operatorname{deg}_{Y, \ldots, Y^{(m)}} P$ is the degree of $P$, then $\mathscr{P}_{y_{0}}$ is situated between the two lines $y=0$ and $y=n$. Furthermore, note that we obtain the same convex hull if we take the points $\left(w^{(j)}, j\right)$. For any edge $e$ of the Newton polygon, we call its slope the number $-\left(i_{1}-i_{2}\right) /\left(j_{1}-j_{2}\right)$ for any pair of distinct points $\left(i_{1}, j_{1}\right),\left(i_{2}, j_{2}\right) \in e$ (note that this is the negative inverse of the usual geometric slope).

We now show how, using the Newton polygon, we can choose the next term $(\bar{\alpha}+\overline{\bar{\alpha}} \sqrt{-1}) x^{\beta}$ in the expansion of $y=y_{0}+(\bar{\alpha}+\overline{\bar{\alpha}} \sqrt{-1}) x^{\beta}+\cdots$. Let $v_{\beta}$ be a vertex of $\mathscr{P}_{y_{0}}$ such that the line $L_{\beta}$ with slope $\beta$ passing through $v_{\beta}$ touches $\mathscr{P}_{y_{0}}$ either just at $v_{\beta}$ or contains an edge $e$ of $\mathscr{P}_{y_{0}}$ descending from $v_{\beta}$. We call $v_{\beta}$ available for the exponent $\beta$. We now fix a certain available vertex $v$ of $\mathscr{P}_{y_{0}}$ (and refer to it as the active vertex) such that $\beta^{(1)}<\beta_{s}$ where $\beta^{(1)}$ is the slope of the edge descending from $v$. Denote by $l$ the ordinate of $v$.

For any $0 \leq j \leq n$, we define the leading polynomial

$$
f_{j} \in \mathbb{Q}\left[\bar{\alpha}_{1}, \ldots, \bar{\alpha}_{s}, \overline{\bar{\alpha}}_{1}, \ldots, \overline{\bar{\alpha}}_{s}, \beta_{1}, \ldots, \beta_{s}, \sqrt{-1}, \beta\right]
$$

as

$$
f_{j}=\sum_{K} \frac{1}{|K| !} l c\left(\left.\frac{\partial^{K} P}{\partial Y^{K}}\right|_{Y=y_{0}}\right)\left(\begin{array}{c}
\beta \\
k_{1}
\end{array}\right) \cdots\left(\begin{array}{l}
\beta \\
k_{j}
\end{array}\right) k_{1} ! \cdots k_{j} !,
$$

where the sum ranges over all $K=\left(k_{1}, \ldots, k_{j}\right)$ such that the weight $w_{K}=$ $\left.\operatorname{deg}_{x} \frac{\partial^{K} P}{\partial Y^{K}}\right|_{Y=y_{0}}-k_{1}-\cdots-k_{j}=w^{(j)}$ and $\left(\begin{array}{c}\beta \\ k_{i}\end{array}\right)=\beta(\beta-1) \cdots\left(\beta-k_{i}+1\right) / k_{i} !$. We 
also define the characteristic polynomial

$$
h_{\beta} \in \mathbb{Q}\left[\bar{\alpha}_{1}, \ldots, \bar{\alpha}_{s}, \overline{\bar{\alpha}}_{1}, \ldots, \overline{\bar{\alpha}}_{s}, \beta_{1}, \ldots, \beta_{s}, \sqrt{-1}, \alpha, \beta\right]
$$

as

$$
h_{\beta}=\sum \alpha^{j} f_{j},
$$

where the sum ranges over all $j$ such that $\left(w^{(j)}, j\right)$ belongs to $L_{\beta}$.

The Newton polygon procedure then has to select the next term $\alpha x^{\beta}$ in such a way that $h_{\beta}(\alpha)=0$. For the same reason as in the classical Newton polygon procedure for expanding an algebraic function as a Puiseux series, $h_{\beta}(\alpha)$ equals the coefficient of the highest term in the expansion of $P\left(y_{0}+\alpha x^{\beta}\right)$ with indeterminate $\alpha$ and $\beta<\beta_{s}$. We now show how to select $\alpha$ and $\beta$.

Two different cases can occur. In the first case $f_{l} \equiv 0$ as a polynomial in $\beta$. Corresponding to this case are two possibilities. For the first possibility we can take any $\beta$ such that $\beta_{s}, \beta^{(2)}>\beta>\beta^{(1)}$, where $\beta^{(2)}$ is the slope of the edge of the polygon $\mathscr{P}_{y_{0}}$ whose lower endpoint is $v$, and an arbitrary $\alpha \neq 0$ for this choice of $\beta$. The second possibility for this case is to take $\beta=\beta^{(1)}$ and $\alpha \neq 0$ such that $h_{\beta}(\alpha)=0$, provided that $h_{\beta}$ has nonzero roots and $\beta^{(1)} \neq-\infty$. In the second case, $f_{l} \not \equiv 0$ and either we take $\beta$ to be a root of $f_{l}(\beta)=0$ such that $\beta_{s}, \beta^{(2)}>\beta>\beta^{(1)}$, provided that such a $\beta$ exists, and an arbitrary $\alpha \neq 0$, or we take $\beta=\beta^{(1)}$ and $\alpha \neq 0$ being a root of $h_{\beta}(\alpha)=0$, provided such an $\alpha$ exists. If the Newton process generates a sequence of $\beta_{i}$ 's such that $\lim \beta_{i}=-\infty$ the formula 1 shows that the corresponding series is a solution of $P(y)=0$. Note that different choices (at any stage) of an active vertex $v$ for $\beta$ or $\alpha$ will lead to all possible generalized series solutions. We illustrate this Newton polygon process with the following

Example. Let $P=x Y^{\prime \prime} Y+Y^{\prime} Y-x Y^{\prime \prime}-Y^{\prime}-x\left(Y^{\prime}\right)^{2}=0$. Figure 1 shows the Newton polygon for this equation. We start by letting $y_{0}=0$. This puts no restrictions on $\beta$ and there are two available vertices: $(-1,2)$ and $(-1,1)$. If we let $(-1,2)$ be the active vertex, then $f_{2} \equiv 0, f_{1}=-\beta^{2}$, and $f_{0}=0$. Since $f_{2} \equiv 0$, we can take $\beta$ such that $\infty>\beta>0$ and $\alpha$ arbitrary or we can let $\beta=\beta^{(1)}=0$ and any $\alpha$ satisfying $h_{\beta}(\alpha)=-\alpha \beta^{2}=-\alpha(0)=0$, i.e. $\alpha$ arbitrary. If we let $(-1,1)$ be the active vertex, then $f_{1}=-\beta^{2} \not \equiv 0$ and $f_{0}=0$. Since $\beta$ must satisfy $0>\beta$ and $\alpha$ must satisfy $h_{\beta}(\alpha)=-\beta^{2} \alpha=0$, we see that letting $(-1,1)$ be an active vertex yields no possible $\beta$. Therefore, we have determined that $y_{0}=\alpha_{0} x^{\beta_{0}}$, with $\beta_{0}>0$ and $\alpha_{0}$ arbitrary or $y_{0}=\alpha_{0}$ with $\alpha_{0}$ arbitrary (note that $y=0$ is a solution of $P(y)=0$ ). We shall now attempt to extend each of these initial segments by adding a term $\alpha x^{\beta}$.

Let $y_{0}=\alpha_{0}$ and replace $y$ by $\alpha_{0}+Y$ in $P(Y)$. We obtain $P\left(\alpha_{0}+Y\right)=$ $\left(\alpha_{0}-1\right) x Y^{\prime \prime}+\left(\alpha_{0}-1\right) Y^{\prime}+x Y^{\prime \prime} Y+Y^{\prime} Y-x\left(Y^{\prime}\right)^{2}$. The Newton polygon for this is shown in Figure 1 (if $\alpha_{0} \neq 1$ ) and in Figure 2 (if $\alpha_{0}=1$ ). Let us assume 


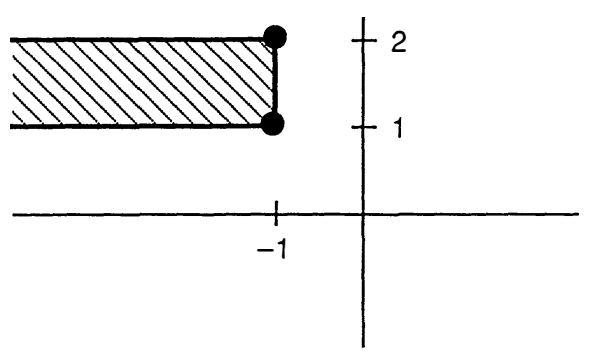

FIGURE 1

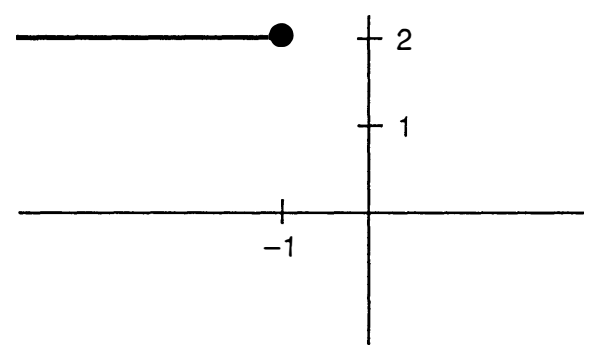

FIGURE 2

that $\alpha_{0} \neq 1$. Since $\beta_{0}=0$, we must have $\beta<0$. This implies that the only available vertex is $(-1,1)$. We have $f_{1}=\left(\alpha_{0}-1\right) \beta^{2}$ and $f_{0}=0$. Therefore $h_{\beta}(\alpha)=\left(\alpha_{0}-1\right) \beta^{2} \alpha$. Since $\alpha_{0} \neq 0$ and $\beta<0$, this has no nonzero root $\alpha$. Now assume $\alpha_{0}=1$. Referring to Figure 2, we see that $(-1,2)$ is an available vertex. We have $f_{2} \equiv 0$, so we can select any $\beta<0$ and any $\alpha$. Therefore, for $y_{0}=\alpha_{0}$ we can extend $y_{0}$ to $y_{1}=\alpha_{0}+\alpha x^{\beta}$ with $\beta<0$ only if $\alpha_{0}=1$ in which case $y_{1}=1+\alpha x^{\beta}$ where $\alpha$ and $\beta$ are arbitrary with $\beta<0$. Note that $y_{0}=\alpha_{0}$ is a solution of $P(y)=0$.

We now let $y_{0}=\alpha_{0} x^{\beta_{0}}, \beta_{0}>0$, and try to extend this to $y_{1}=\alpha_{0} x^{\beta_{0}}+\alpha x^{\beta}$, $\beta_{0}>\beta$. We have

$$
\begin{aligned}
P\left(y_{0}+Y\right)= & P\left(\alpha_{0} x^{\beta_{0}}+Y\right)=-\alpha_{0} \beta_{0}^{2} x^{\beta_{0}-1}+\left(\alpha_{0} x^{\beta_{0}+1}-x\right) Y^{\prime \prime} \\
& +\left(\alpha_{0} x^{\beta_{0}}-1-2 \alpha_{0} \beta_{0} x^{\beta_{0}}\right) Y^{\prime}+\left(\alpha_{0} \beta_{0}^{2} x^{\beta_{0}-1}\right) Y \\
& +Y^{\prime \prime} Y+Y Y^{\prime}-x\left(Y^{\prime}\right)^{2} .
\end{aligned}
$$

Figure 3 shows the Newton polygon for this differential polynomial. Note that the coefficient of $Y^{\prime \prime}$ is nonzero since $\beta_{0}+1>1$, the coefficient of $Y^{\prime}$ is nonzero since $\beta_{0}>1$, and the coefficient of $Y$ is nonzero since $\beta_{0}>0$ and $\alpha_{0} \beta_{0}^{2} \neq 0$. The only available vertices are $\left(\beta_{0}-1,1\right)$ and $\left(\beta_{0}-1,0\right)$. Note that if we let $\left(\beta_{0}-1,0\right)$ be the active vertex, then $f_{0}=-\alpha_{0} \beta_{0}^{2} \neq 0$ and $h_{\beta}(\alpha)=-\alpha_{0} \beta_{0}^{2}$, which has no roots (in general, letting a vertex of the form $\left(w_{K}, 0\right)$ be an active vertex leads nowhere). Therefore we let $\left(\beta_{0}-1,1\right)$ be the 


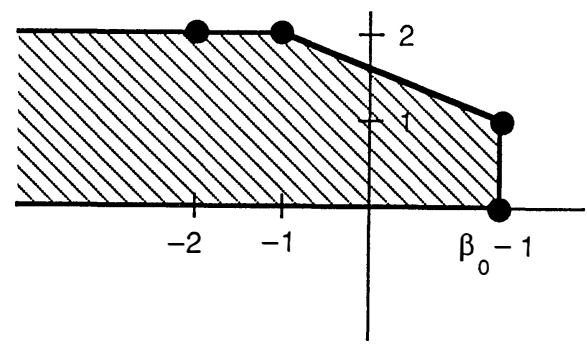

FIGURE 3

active vertex. We have $f_{1}=\alpha_{0}\left[\beta_{0}^{2}-2 \beta_{0} \beta+\beta^{2}\right]=\alpha_{0}\left(\beta-\beta_{0}\right)^{2}$ and $f_{0}=-\alpha_{0} \beta_{0}^{2}$. We have $f_{1} \not \equiv 0$ and we cannot take $\beta$ as a root of $f_{1}=0$. Therefore, we must let $\beta=0$. We then have $h_{\beta}(\alpha)=\alpha_{0} \beta_{0}^{2} \alpha-\alpha_{0} \beta_{0}^{2}$, so $\alpha=1$. Therefore, if we try to extend $y_{0}=\alpha_{0} x^{\beta_{0}}, \beta_{0}>0$, to $y_{1}=\alpha_{0} x^{\beta_{0}}+\alpha x^{\beta}$, we must have $\beta=0$ and $\alpha=1$.

In summary, we see that after applying the Newton procedure twice, we have generated the following possible initial segments: $1+\alpha x^{\beta}$, with $\beta<0$ and $\alpha$ arbitrary; $\alpha$, with $\alpha$ arbitrary; and $\alpha x^{\beta}+1$, with $\beta>0$ and $\alpha$ arbitrary. The reader should show that each of these is actually a solution of $P(Y)=0$ and that none of these can be further extended.

The Newton polygon procedure described above allows us to obtain an extension $y_{1}=y_{0}+\alpha x^{\beta}$ of the given initial segment $y_{0}$. We now construct the new Newton polygon $\mathscr{P}_{y_{1}}$ corresponding to $y_{1}$. By Taylor's formula, we have

$$
\begin{aligned}
P^{\left(y_{1}\right)} & =P\left(y_{1}+Y\right)=\left.\sum_{K} \frac{1}{|K| !} \frac{\partial^{K} P}{\partial Y^{K}}\right|_{Y=y_{1}} Y^{K} \\
& =\left.\sum_{K} \frac{1}{|K| !} \sum_{K^{(1)}} \frac{1}{\left|K^{(1)}\right| !} \frac{\partial^{K+K^{(1)}} P}{\partial Y^{K+K^{(1)}}}\right|_{Y=y_{0}}\left(\alpha x^{\beta}\right)^{K^{(1)}} Y^{K} .
\end{aligned}
$$

Hence, the degree in $x$ of the coefficient of $Y^{K}$ in $P^{\left(y_{1}\right)}$ (where $K=\left(k_{1}, \ldots\right.$, $\left.k_{j}\right)$ ) is at most

$$
\max _{K^{(1)}=\left(k_{1}^{(1)}, \ldots, k_{j_{1}}^{(1)}\right)}\left\{\left.\operatorname{deg}_{x} \frac{\partial^{K+K^{(1)} P}}{\partial Y^{K+K^{(1)}}}\right|_{Y=y_{0}}+j_{1} \beta-k_{1}^{(1)}-\cdots-k_{j_{1}}^{(1)}\right\} .
$$

The following lemma compares $\mathscr{P}_{y_{1}}$ to $\mathscr{P}_{y_{0}}$.

Lemma 2.1. Let $\left(w^{(l)}, l\right)$ be the active vertex for $\beta$ in $\mathscr{P}_{y_{0}}$ and let $\mathscr{P}_{y_{1}}$ be the new Newton polygon corresponding to $y_{1}=y_{0}+\alpha x^{\beta}$.

(i) Any point $(p, j)$ such that $p+j \beta>w^{(l)}+l \beta$ does not belong to $\mathscr{P}_{y_{1}}$. 
(ii) If $(p, j) \in \mathscr{P}_{y_{0}}$ lies on the boundary of $\mathscr{P}_{y_{0}}$ and $j \geq l$, then $(p, j) \in$ $\mathscr{P}_{y_{1}}$ and the leading polynomial $f_{j}$ is the same for $\mathscr{P}_{y_{1}}$ as for $\mathscr{P}_{y_{0}}$.

(iii) $\left(w^{(l)}+l \beta, 0\right)$ does not belong to $\mathscr{P}_{y_{1}}$. Therefore $\mathscr{P}_{y_{1}}$ contains at least one edge with a slope less than $\beta$ and so, at the next step, we can pick out an active vertex with ordinate less than or equal to $l$.

Proof. (i) First note that

$$
\begin{aligned}
\operatorname{deg}_{x} & \left.\frac{\partial^{K+K^{(1)}} P}{\partial Y^{K+K^{(1)}}}\right|_{Y=y_{0}}+j_{1} \beta-k_{1}^{(1)}-\cdots-k_{j_{1}}^{(1)} \leq w^{\left(j+j_{1}\right)} \\
& +\left(k_{1}+\cdots+k_{j}\right)+j_{1} \beta \leq w^{(l)}+l \beta+\left(k_{1}+\cdots+k_{j}\right)-j \beta .
\end{aligned}
$$

This last inequality is because $w^{\left(j+j_{1}\right)}+\left(j+j_{1}\right) \beta \leq w^{(l)}+l \beta$ by the choice of $L_{\beta}$. If $(p, j) \in \mathscr{P}_{y_{1}}$ then $p=\max _{K=\left(k_{1}, \ldots, k_{1}\right)} w_{K}$, where $w_{K}$ is the weight of the term $P_{K} Y^{K}$ in $P\left(y_{1}+Y\right)$. Therefore

$$
p \leq\left.\operatorname{deg}_{x} \frac{\partial^{K+K^{(1)}} P}{\partial Y^{K+K^{(1)}}}\right|_{Y=y_{0}}+j_{1} \beta-k_{1}^{(1)}-\cdots-k_{j_{1}}^{(1)}
$$

for an appropriate $K^{(1)}$ and so, by the above, $p \leq w^{(l)}+l \beta-j \beta$. This contradicts the assumption that $p+j \beta>w^{(l)}+l \beta$ and proves (i).

(ii) Fix some $K=\left(k_{1}, \ldots, k_{j}\right)$. If $w_{K}=w^{(j)}$, we obtain, from formula (2) (taking $K^{(1)}=0$ ), that the term

$$
\left.\frac{1}{|K| !} \frac{\partial^{K} P}{\partial Y^{K}}\right|_{Y=y_{0}}\left(\begin{array}{c}
\beta \\
k_{1}
\end{array}\right) \cdots\left(\begin{array}{c}
\beta \\
k_{j}
\end{array}\right) k_{1} ! \cdots k_{j} !
$$

gives a contribution to $f_{j}$. If $K^{(1)}=\left(k_{1}^{(1)}, \ldots, k_{j_{1}}^{(1)}\right) \not \equiv 0$, then the term

$$
\left.\frac{\partial^{K+K^{(1)}} P}{\partial Y^{K+K^{(1)}}}\right|_{Y=y_{0}}\left(\alpha x^{\beta}\right)^{K^{(1)}}
$$

does not contribute to $f_{j}$. To see this note first that

$$
\left.\operatorname{deg}_{x} \frac{\partial^{K+K^{(1)}} P}{\partial Y^{K+K^{(1)}}}\right|_{Y=y_{0}}+\beta_{j_{1}}-k_{1}^{(1)}-\cdots-k_{j_{1}}^{(1)} \leq w^{\left(j+j_{1}\right)}+\beta_{j_{1}}+k_{1}+\cdots+k_{j} .
$$

Since any edge of $\mathscr{P}_{y_{0}}$ above the line $Y=l$ has slope $>\beta$, then we have $w^{\left(j+j_{1}\right)}+\beta\left(j+j_{1}\right)<\omega^{(j)}+\beta_{j}$. We therefore have

$$
\left.\operatorname{deg}_{x} \frac{\partial^{K+K^{(1)}} P}{\partial Y^{K+K^{(1)}}}\right|_{Y=y_{0}}+\beta_{j_{1}}-k_{1}^{(1)}-\cdots-k_{j_{1}}^{(1)}<w^{(j)}+k_{1}+\cdots+k_{j} ;
$$


and so if $K^{(1)} \neq 0$, the term

$$
\left.\frac{\partial^{K+K^{(1)}} P}{\partial Y^{K+K^{(1)}}}\right|_{y=y_{0}}\left(\alpha x^{\beta}\right)^{K^{(1)}}
$$

does not contribute to $f_{j}$. Thus $f_{j}$, and by the same token, $(p, j)$, do not change in $\mathscr{P}_{y_{1}}$

(iii) Consider the point $\left(w^{(l)}+l \beta, 0\right)$. To this point corresponds the coefficient (put $K=0$ in (2))

$$
\sum_{K^{(1)}=\left(k_{1}^{(1)}, \ldots, k_{j_{1}}^{(1)}\right)} \frac{1}{\left|K^{(1)}\right| !} l c\left(\left.\frac{\partial^{K^{(1)}} P}{\partial Y^{K^{(1)}}}\right|_{y_{0}=Y}\right) \alpha^{j_{1}}\left(\begin{array}{c}
\beta \\
k_{1}^{(1)}
\end{array}\right) \cdots\left(\begin{array}{c}
\beta \\
k_{j_{1}}^{(1)}
\end{array}\right) k_{1}^{(1)} ! \cdots k_{j_{1}}^{(1)} !,
$$

where the summation ranges over all $K^{(1)}$ such that

$$
\operatorname{deg}\left(\left.\frac{\partial^{K^{(1)}} P}{\partial Y^{K^{(1)}}}\right|_{y_{0}=Y}\right)+\beta_{j_{1}}-k_{1}^{(1)}-\cdots-k_{j_{1}}^{(1)}=w^{(l)}+l \beta .
$$

In particular, for $k_{1}$ we have $w^{\left(j_{1}\right)}+\beta_{j_{1}}=w^{(l)}+l \beta$, and this proves that the coefficient (3) equals $h_{\beta}(\alpha)$, which is 0 . This shows that the point $\left(w^{(l)}+l \beta, 0\right)$ does not belong to $\mathscr{P}_{y_{1}}$. The second part of (iii) follows from this.

The case when at the next step we can take the same $v$ as an active vertex can only happen if the edge descending from the endpoint $v$ in the polygon $\mathscr{P}_{y_{1}}$ has a slope less than $\beta$. The following lemma tells us when this can continue to happen.

Lemma 2.2. Let $y=\sum_{i=0}^{\infty} \alpha_{i} x^{\beta_{\imath}}$ be generated by the Newton polygon process as above and assume $y \in \Omega$. If, in this process, a vertex $v$ with ordinate $l$ becomes available and stays available at each step thereafter, then $\partial^{K} P /\left.\partial Y^{K}\right|_{Y=y}=0$ for all $K=\left(k_{1}, \ldots, k_{j}\right)$ for which $j<l$.

Proof. Assume that after the $t$ th step the vertex $v=\left(w^{(l)}, l\right)$ becomes active and stays active. For any $s$ and vertex $(p, j)$ of the $s+t$ th Newton polygon with $j \leq l$ we have $p+\beta_{t+s} j \leq w^{(l)}+\beta_{t+s} l$, so this point lies to the left of $X+\beta_{t+s} Y=w^{(l)}+\beta_{t+s} l$ and between $Y=0$ and $Y=l$. If $\partial^{K} P /\left.\partial Y^{K}\right|_{Y=y}=\alpha x^{\beta}+$ terms with smaller exponent, $\alpha \neq 0$, then by Lemma 1.6, $\partial^{K} P /\left.\partial Y^{K}\right|_{Y=y_{t+s}}=\alpha x^{\beta}+$ terms with smaller exponents for sufficiently large $s$. Since we are assuming that $\lim _{s \rightarrow \infty} \beta_{t+s}=-\infty$, formula (1) shows that this would produce a point in the Newton polygon to the right of $X+\beta_{t+s} Y=\omega^{(l)}+\beta_{t+s} l$. Therefore, $\partial^{K} P /\left.\partial Y^{K}\right|_{Y=y}=0$.

We end this section by noting that a Newton polygon type process has been previously used by several authors (e.g. [RA78 and DE82]) to find solutions of 
linear differential equations and to find solutions of some nonlinear first order differential equations (e.g. [HI76]).

\section{Stabilization of the Newton POlygon PROCESS}

We begin with the following definition. A solution $y=\sum\left(\overline{\alpha_{1}}+\sqrt{-1} \overline{\bar{\alpha}}_{i}\right) x^{\beta_{\imath}} \in$ $\Omega$ of $P(Y)=0$ is called stabilizable for $P$ if after some steps of the Newton polygon process we come to a Newton polygon $\mathscr{P}_{y_{0}}$ corresponding to an initial segment $y_{0}=\sum_{1 \leq i \leq s}\left(\overline{\alpha_{i}}+\sqrt{-1} \overline{\bar{\alpha}}_{i}\right) x^{\beta_{\imath}}$ with active vertex $v$ having ordinate 1 and only the edge descending from the vertex $v$ has a slope less than $\beta_{s}$. Furthermore, we require that $\beta_{s}$ is not greater than any real root of the polynomial $f_{1}(\beta)$. We also describe this situation as $y$ is stable for $P$ after $y_{0}$. We make the following two remarks. The first is that $f_{1} \not \equiv 0$ as a polynomial in $\beta$. Indeed,

$$
f_{1}(\beta)=\sum_{q} \frac{1}{q !} \gamma_{q} \beta(\beta-1) \cdots(\beta-q+1),
$$

where $\gamma_{q}=l c\left(\partial P /\left.\partial Y^{(q)}\right|_{y_{0}=Y}\right)$ and the summation ranges over all $q$ such that the weight

$$
\operatorname{deg}_{x}\left(\left.\frac{\partial P}{\partial Y^{(q)}}\right|_{y_{0}=Y}\right)-q=w^{(1)}
$$

which is the abscissa of the point $v$. Obviously, $f_{1} \not \equiv 0$.

The second remark is that after stabilizing there is a unique possibility for choosing the next term $\alpha x^{\beta}$ in the expansion $y=y_{0}+\alpha x^{\beta}+\cdots$; namely $\beta$ is the slope of the edge $e$ of the Newton polygon descending from $v$ and $\alpha$ satisfies the equation $h_{\beta}(\alpha)=f_{1}(\beta) \alpha+l c\left(P\left(y_{0}\right)\right)=0$. Therefore the Newton polygon process, after stabilizing, can be continued uniquely and correctly and leads to the unique solution $y \in \Omega$ of $P(Y)=0$ with initial segment $y_{0}$, provided that the slopes tend to $-\infty$. If the latter does not hold, we get no solution with initial segment $y_{0}$ (by Theorem 1.1). The next lemma states that although a solution $y$ (with an infinite number of terms) of $P=0$ may not be stabilizable for $P, y$ is stabilizable for some partial derivative of $P$.

Lemma 3.1. For any solution $y=\sum\left(\bar{\alpha}_{i}+\sqrt{-1} \overline{\bar{\alpha}}_{i}\right) x^{\beta_{\imath}} \in \Omega$ of $P(Y)=0$ (provided that the series has an infinite number of terms so $\lim \beta_{i}=-\infty$ by Theorem 1.1) consider an index I for which

$$
\left.\frac{\partial^{I} P}{\partial Y^{I}}\right|_{Y=y}=0, \quad \frac{\partial^{I} P}{\partial Y^{I}} \not \equiv 0, \quad \text { and } \operatorname{deg}_{Y, Y^{(1)}, \ldots, Y^{(m)}}\left(\frac{\partial^{I} P}{\partial Y^{I}}\right)
$$

is the least possible. Then $y$ is a stabilizable solution for $\frac{\partial^{I} P}{\partial Y^{I}}$.

Proof. First we show that in the Newton polygon process for expanding $y$ as a solution of $\partial^{I} P / \partial Y^{I}=0$, we eventually come to an active point with ordinate 1. Assume the contrary, then starting from some step, a certain vertex $v$ with ordinate $l>1$ would become and stay an active point. By virtue of Lemma 2.2, 
$\partial^{I+K} P /\left.\partial Y^{I+K}\right|_{y=Y}=0$ for all $K=\left(k_{1}, \ldots, k_{j}\right)$ such that $j<l$. For some $K=\left(k_{1}, \ldots, k_{j}\right)$ we have $\partial^{I+K} P / \partial Y^{I+K} \not \equiv 0$, hence the multi-index $I+K$ satisfies the conditions of the lemma, contradicting the choice of $I$. Thus, we have proved that a certain vertex $v$ with ordinate 1 eventually would become and stay an active point.

Continuing the Newton polygon process we would eventually have that $\beta_{0}$ would be less than any real root of the polynomial $f_{1}(\beta)$ by Theorem 1.1. This means that $y$ is is a stabilizable solution for $\frac{\partial^{I} P}{\partial Y^{T}}$ and completes the proof of the lemma.

Corollary. For any solution

$$
y=\sum\left(\overline{\alpha_{i}}+\sqrt{-1} \overline{\bar{\alpha}}_{i}\right) x^{\beta_{i}}
$$

of $P(Y)$, the exponents $\beta_{0}, \beta_{1}, \ldots$ generate a finite $\mathbb{Z}$ module.

Proof. Any solution $y$ of $P(y)=0$ is stabilizable for $\partial^{I} P / \partial Y^{I}$ for some $I$. If $y$ is stable for $\partial^{I} P / \partial Y^{I}$ after $y_{0}=\sum_{0 \leq i \leq s}\left(\bar{\alpha}_{i}+\sqrt{-1} \overline{\bar{\alpha}}_{i}\right) x^{\beta_{\imath}}$, then all the exponents $\beta_{i}$ lie in the $\mathbb{Z}$-module generated by $1, \beta_{0}, \ldots, \beta_{s}$.

Note that the set $\Omega^{\mathrm{fin}}=\left\{\sum\left(\bar{\alpha}_{i}+\sqrt{-1} \overline{\bar{\alpha}}_{i}\right) x^{\beta_{\imath}} \in \Omega \mid\left\{\beta_{i}\right\}\right.$ generate a finite $\mathbb{Z}$ module $\}$ forms a differential field.

\section{ENUMERATING THE GENERALIZED SERIES SOLUTIONS OF AN ORDINARY DIFFERENTIAL EQUATION}

The Newton polygon process described above can be used to enumerate (in a sense made precise below) the generalized series solutions of a differential equation $P=0$, where $P$ is the same as the beginning of $\S 2$. If we consider an initial segment $y_{0}=\sum_{1 \leq i \leq s}\left(\bar{\alpha}_{i}+\sqrt{-1} \overline{\bar{\alpha}}_{i}\right) x^{\beta_{\imath}}$ of $P=0$ where we are thinking of the $\bar{\alpha}_{i}, \overline{\bar{\alpha}}_{i}$, and $\beta_{i}$ as real parameters, then the next iteration of the Newton polygon procedure used to extend $y_{0}$ to $y_{0}+(\bar{\alpha}+\sqrt{-1} \overline{\bar{\alpha}}) x^{\beta}$ will place restrictions on these parameters. These restrictions can be expressed as a certain quantifier-free formula $\varphi\left(\bar{\alpha}_{1}, \ldots, \bar{\alpha}_{s}, \overline{\bar{\alpha}}_{1}, \ldots, \overline{\bar{\alpha}}_{s}, \beta_{1}, \ldots, \beta_{s}, \bar{\alpha}, \overline{\bar{\alpha}}, \beta\right)$ in the first-order language of ordered fields. We consider the validity of such formulas in the first-order theory of real closed fields (sometimes called the Tarski algebra) and we shall refer to these as Tarski algebra formulas. It is well known that the Tarski algebra is decidable [TA51, CO75, GR88]. The rest of this section is devoted to the proof of the following:

Theorem 4.1. Let an ordinary differential equation $P=0$ be given. There is a procedure that enumerates all the generalized series solutions of $P=0$ in the following sense. It enumerates a succession of consistent Tarski algebra formulas

$$
\Psi\left(\overline{\alpha_{1}}, \ldots, \overline{\alpha_{s_{j}}}, \overline{\bar{\alpha}}_{1}, \ldots, \overline{\bar{\alpha}}_{s_{j}}, \beta_{1}, \ldots, \beta_{s_{j}}\right)
$$

with parameters and ordinary differential polynomials $P_{j} \in Q[x]\{Y\}$ such that for each $j$ and any $\bar{\alpha}_{1}^{(0)}, \ldots, \overline{\bar{\alpha}}_{s_{j}}^{(0)}, \beta_{1}^{(0)}, \ldots, \beta_{s_{j}}^{(0)} \in \mathbb{R}$ satisfying this formula, 
$y_{0}=\sum_{1 \leq i \leq s_{j}}\left(\bar{\alpha}_{i}^{(0)}+\sqrt{-1} \overline{\bar{\alpha}}_{i}^{(0)}\right) x^{\beta_{i}^{(0)}}$ is an initial segment of an expansion of solution $y=y_{0}+\cdots \in \Omega$ of the equation $P_{j}=0$, provided that the slopes in the expansion tend to $-\infty$. If the latter holds this solution $y$ is stable for $P_{j}$ after producing the initial segment $y_{0}$ via the Newton polygon procedure (so $y$ is uniquely defined from $y_{0}$ on) and $P(y)=0$. Any generalized series solution of $P(y)=0$ appears in this way.

In the procedure that we now describe, the Tarski algebra formulas are generated when we attempt to extend an initial segment $y_{0}=\sum_{1 \leq i \leq s}\left(\bar{\alpha}_{i}+\sqrt{-1} \overline{\bar{\alpha}}_{i}\right) x^{\beta_{\imath}}$. We will proceed by induction on $s$, the number of terms in the initial segment $y_{0}$. So, we are given a set of parameters $\bar{\alpha}_{1}, \ldots, \bar{\alpha}_{s}, \overline{\bar{\alpha}}_{1}, \ldots, \overline{\bar{\alpha}}_{s}, \beta_{1}, \ldots, \beta_{s}$ and a certain Tarski algebra formula $\varphi\left(\bar{\alpha}_{1}, \ldots, \bar{\alpha}_{s}, \overline{\bar{\alpha}}_{1}, \ldots, \overline{\bar{\alpha}}_{s}, \beta_{1}, \ldots, \beta_{s}\right)$. At the base of the induction $s=0$ and the formula $\varphi$ is $(0=0)$. In addition to $\varphi$ we will also be given an equation $Q=0$, an inequality $Q_{0} \neq 0$, a Newton polygon $\mathscr{P}_{y_{0}}^{(Q)}$ for the equation $Q=0$ corresponding to the initial segment $y_{0}$, and lastly an active vertex at the previous step of the Newton polygon process. Here $Q(Y), Q_{0}(Y)$ are differential polynomials such that $Q_{0} \prec Q$ where the latter relations mean that either the order of $Q_{0}$ is less than the order of $Q$ or that they both have the same order $m$ and the degree of $Q_{0}$ in $Y^{(m)}$ is less than the degree of $Q$ in $Y^{(m)}$. When $s=0$, $Q=P$ and $Q_{0}=1$. The polygon $\mathscr{P}_{y_{0}}^{(Q)}$ is represented by specifying the integers $0 \leq t_{1}<\cdots<t_{u} \leq n$ and a subset of them $0 \leq t_{1}^{\prime}<\cdots<t_{u^{\prime}}^{\prime} \leq n$ such that the ordinates of the vertices belonging to the boundary of $\mathscr{P}_{y_{0}}^{(Q)}$ are exactly $t_{1}, \ldots, t_{u}$ and the endpoints of the edges of $\mathscr{P}_{y_{0}}^{(Q)}$ have exactly the ordinates $t_{1}^{\prime}, \ldots, t_{u^{\prime}}^{\prime}$. For each $t_{j}$ we are given the abscissa $p_{j}$ of the corresponding vertex, where $p_{j}=p_{j}^{(1)} \beta_{1}+\cdots+p_{j}^{(s)} \beta_{s}+p_{j}^{(0)}$, where $p_{j}^{(1)}, \ldots, p_{j}^{(s)}, p_{j}^{(0)} \in \mathbb{Z}$, and $p_{j}^{(1)}, \ldots, p_{j}^{(s)} \geq 0$. The algorithm also produces the leading polynomial $f_{t} \in Q\left[\overline{\alpha_{1}}, \ldots, \bar{\alpha}_{s}, \overline{\bar{\alpha}}_{1}, \ldots, \overline{\bar{\alpha}}_{s}, \beta_{1}, \ldots, \beta_{s}, \sqrt{-1}\right]$ corresponding to the vertex $\left(p_{j}, t_{j}\right)$ of $\mathscr{P}_{y_{0}}^{(Q)}$. The active vertex at the previous step is specified by indicating some $t_{j}^{\prime}$.

We now describe the next step of the enumeration algorithm, during which the next term $\alpha x^{\beta}$ of $y=y_{0}+\alpha x^{\beta}+\cdots$ will be produced. Firstly, we select an active vertex $v$ with ordinate $l=t_{j^{\prime}}^{\prime}$ less than or equal to $t_{j}^{\prime}$. We then choose the exponent $\beta$ according to the following possibilities (cf. §2). Let $f_{l}=$ $\sum_{0 \leq j \leq j_{0}} f_{l}^{(j)} \beta^{j}$ be a leading polynomial, where $f_{l}^{(j)} \in \mathbb{Q}\left[\bar{\alpha}_{1}, \ldots, \bar{\alpha}_{s}, \overline{\bar{\alpha}}_{1}, \ldots\right.$, $\left.\overline{\bar{\alpha}}_{s}, \beta_{1}, \ldots, \beta_{s}, \sqrt{-1}\right]$. The first case is when the line with the next slope $\beta$ passing through the node $v$ lies strictly outside $P_{y_{0}}^{(Q)}$ and strictly between two edges of the Newton polygon, with slopes $\delta_{1}>\delta_{2}$ respectively, adjacent to $v$. We then add to the formula $\varphi$ the following conditions: $\delta_{1}>\beta>\delta_{2}, \beta_{s}>\beta$, $f_{l}(\beta)=0$ (and in this case $\bar{\alpha}$ and $\overline{\bar{\alpha}}$ are arbitrary). In the second case, when 
$\beta=\delta_{2}$, the algorithm adds to $\varphi$ this latter equality as well as the condition $\beta_{s}>\beta$ and does the following. The algorithm produces the characteristic polynomial $h_{\delta_{2}} \in Q\left[\overline{\alpha_{1}}, \ldots, \overline{\alpha_{m}}, \overline{\bar{\alpha}}_{1}, \ldots, \overline{\overline{\alpha_{m}}}, \beta_{1}, \ldots, \beta_{m}, \sqrt{-1}, \alpha\right]$, (see above) corresponding to the edge with slope $\delta_{2}$ and with endpoint $v$. Note that $h_{\delta_{2}}$ involves $t_{j}, p_{j}$ and $f_{j}$. The expression $\bar{\alpha}+\sqrt{-1} \overline{\bar{\alpha}}$ is then substituted for $\alpha$ in $h_{\delta_{2}}$, and we write

$$
h_{\delta_{2}}(\bar{\alpha}+\sqrt{-1} \overline{\bar{\alpha}})=\bar{h}_{\delta_{2}}(\bar{\alpha}, \overline{\bar{\alpha}})+\sqrt{-1 \bar{h}_{\delta_{2}}}(\bar{\alpha}, \overline{\bar{\alpha}}) .
$$

Finally, we add to the formula $\varphi$ the equations $\overline{h_{\delta_{2}}}(\bar{\alpha}, \overline{\bar{\alpha}})=\overline{\overline{h_{\delta_{2}}}}(\bar{\alpha}, \overline{\bar{\alpha}})=0$. The resulting Tarski formula is denoted by $\hat{\varphi}$. We can check whether such $\beta$, $\delta_{1}>\beta>\delta_{2}$ exists. If not, we do not consider $v$ as an available node. We wish to continue this process and our initial segment stabilizes. Since an initial segment may not stabilize for $Q=0$ but only for some derivative $\partial^{K} Q / \partial Y^{K}$ we are forced to consider various branchings in our procedure. Consider all the differential polynomials $\partial^{K} Q / \partial Y^{K}$ for all $K=\left(k_{1}, \ldots, k_{j}\right)$ with $j<l$. Observe that $\partial^{K} Q / \partial Y^{K} \prec Q$. Now the process branches in two ways.

(A) First, assume $l>1$ and continue the Newton polygon process applying it to $Q$. At this point we have an initial segment

$$
\overline{y_{0}}=\sum_{1 \leq j \leq s}\left(\overline{\alpha_{j}}+\sqrt{-1} \overline{\bar{\alpha}}_{j}\right) x^{\beta_{i}}+(\bar{\alpha}+\overline{\bar{\alpha}} \sqrt{-1}) x^{\beta},
$$

the same equation $Q=0$, and the same inequality $Q_{0} \neq 0$. And we yield a new Newton polygon $\mathscr{P}_{\overline{y_{0}}}^{(Q)}$ in the following way. Recall (see (1)) that

$$
Q=\left.\sum_{K} \frac{1}{|K| !} \frac{\partial^{K} Q}{\partial Y^{K}}\right|_{\overline{y_{0}}=Y} Y^{K}=\sum_{\bar{K}} \bar{\rho}_{\bar{K}} x^{k_{0}} Y^{K},
$$

where $\bar{K}=\left(k_{0}, K\right)=\left(k_{0}, k_{1}, \ldots, k_{j}\right), \bar{\rho}_{\bar{K}} \in Q\left[\overline{\alpha_{1}}, \ldots, \bar{\alpha}_{s}, \overline{\bar{\alpha}}_{1}, \ldots, \overline{\bar{\alpha}}_{s}, \beta_{1}\right.$, $\left.\ldots, \beta_{s}, \sqrt{-1}, \bar{\alpha}, \overline{\bar{\alpha}}, \beta\right], k_{0}=\sum \kappa_{j} \beta_{j}+\kappa_{0}$, where $0 \leq \kappa_{1}, \ldots, \kappa_{s} \in \mathbb{Z}, \kappa_{0} \in$ $\mathbb{Z}, 0 \leq k_{1}, \ldots, k_{j} \in \mathbb{Z}$. Now we consider all the finite number of possible shapes of $\mathscr{P}_{\overline{y_{0}}}^{(Q)}$. A shape means two lists of integers $0 \leq \overline{t_{1}} \leq \cdots \leq \overline{t_{u}}<l$, $0 \leq \bar{t}_{1}^{\prime}<\cdots<\bar{t}_{u^{\prime}}^{\prime}<l$ such that $\bar{t}_{1}^{\prime}, \ldots, \bar{t}_{u^{\prime}}^{\prime}$ are the ordinates of the extremal vertices, with ordinates less than $l$, of $\mathscr{P}_{\overline{y_{0}}}(Q)$ (recall that the vertices of the Newton polygon with ordinates not less than $l$ do not change), and $\overline{t_{1}}, \ldots, \overline{t_{u}}$ are all the vertices, with ordinates less than $l$, of $\mathscr{P} \frac{(Q)}{\bar{y}_{0}}$. Observe that the shapes can be expressed in terms of a quantifier-free Tarski algebra formula in the parameters $\bar{\alpha}_{1}, \ldots, \bar{\alpha}_{s}, \overline{\bar{\alpha}}_{1}, \ldots, \overline{\bar{\alpha}}_{s}, \beta_{1}, \ldots, \beta_{s}, \bar{\alpha}, \overline{\bar{\alpha}}, \beta$ (actually, it is a formula of linear programming, i. e. all the inequalities are linear).

(B) Second, provided again that $l>1$, we consider a system of differential equations $\mathscr{S}=\{Q=0\} \cup\left\{\partial^{K} Q / \partial Y^{K}=0 \mid\right.$ for all $\left.K=\left(k_{1}, \ldots, k_{j}\right), j<l\right\}$. 
We apply the reduction process from [SE56] to the system $\mathscr{S} \cup\left\{Q_{0} \neq 0\right\}$ and obtain as a result that the latter system is equivalent (over a differential closure) to a disjunction of the following form: $\bigvee_{i}\left(Q_{i}=0, Q_{i_{0}} \neq 0\right)$, where $Q_{i} \prec \partial^{K} Q / \partial Y^{K} \preceq Q$, for $K=\left(k_{1}, \ldots, k_{j}\right), j<l$, and $Q_{i_{0}} \prec Q_{i}$. For any $i$ we consider the system $Q_{i}=0, Q_{i_{0}} \neq 0$ as a new starting point for the Newton polygon process. We consider $\left(\bar{\alpha}_{1}+\overline{\bar{\alpha}}_{1} \sqrt{-1}\right) x^{\beta_{1}}$ as the first term, then substituting it in $Q_{i}$ gives the next Newton polygon and Tarski algebra formula; if it is consistent, we substitute the next term $\left(\bar{\alpha}_{2}+\sqrt{-1 \bar{\alpha}_{2}}\right) x^{\beta_{2}}$, etc., up to the term $\left(\bar{\alpha}_{s}+\sqrt{-1 \bar{\alpha}_{s}}\right) x^{\beta_{s}}$. After this we start to apply the recursive process, under description, to the system $Q_{i}=0, Q_{i_{0}} \neq 0$, the constructed Newton polygon and the Tarski algebra formula.

Now assume that the ordinate of the active vertex $v$ is 1 . Fix some natural number $N$ and consider the following condition. Recall that $f_{1}(\boldsymbol{Z}) \in$ $Q\left[\overline{\alpha_{1}}, \ldots, \bar{\alpha}_{s}, \overline{\bar{\alpha}}_{1}, \ldots, \overline{\bar{\alpha}}_{s}, \beta_{1}, \ldots, \beta_{s}, \sqrt{-1}\right][Z]$ is the leading polynomial corresponding to the active node $v$ and suppose that $\operatorname{deg}_{Z} f_{1}=l$ for some $l$ and $f_{1}(Z)=a_{l} Z^{l}+\cdots+a_{0}, a_{l} \neq 0$. The condition we are considering is that the Newton polygon process applied to $Q$ correctly gives, after $N$ steps,

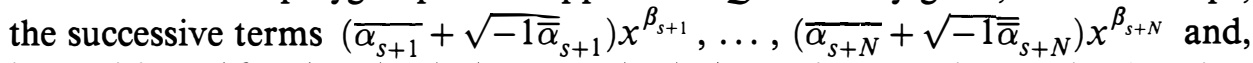
in addition, $\left|\beta_{s+N}\right|>\left|\alpha_{0} / \alpha_{l}\right|+\cdots+\left|\alpha_{l} / \alpha_{l}\right|$. This latter inequality just implies that $\beta_{s+N}$ is less than any real root of the polynomial $f_{1}$. The described condition can be represented by a quantifier-free Tarski algebra formula $\psi_{N}$ in $\overline{\alpha_{j}}, \overline{\bar{\alpha}}_{j}, \beta_{j}, 1 \leq j \leq s+N$ (cf. above). Note that for any solution $y$ of $Q(Y)=0$ and some initial segment $y_{0}$ for which the next active vertex $v$ has ordinate 1 , we know by Theorem 1.1 that an $N$ exists such that the next $N$ terms of this solution satisfy such a Tarski algebra formula.

At this point we have generated, for a fixed $N$, a Tarski formula that states that a segment of length $s+N$ is an initial segment of a possible solution $y$ of $P=0$ and is stable for some equation $Q=0$ provided that the slopes after stabilization tend to $-\infty$. In the process we have generated an auxiliary equation $Q_{0} \neq 0$ which must be satisfied to guarantee that $y$ satisfies $P=0$. To see if $Q_{0}(y) \neq 0$, we carry out the following subprocedure that looks for an exponent $M_{0}$ such that $Q_{0}(y) \neq 0\left(\bmod x^{M_{0}}\right)$. For the $N$ fixed above, we fix some $N_{0}$ and some term of $Q_{0}$ of the form $x^{l_{0}} y^{\left(l_{1}\right)} \cdots y^{\left(l_{s}\right)}$, namely, some term of its expansion of the form $x^{l_{0}} x^{\beta_{J_{1}}-l_{1}} \ldots x^{\beta_{J_{s}}-l_{s}}$ where $0 \leq j_{1}, \ldots, j_{s} \leq$ $N_{0}$. The subprocedure looks over all $N_{0}, j_{1}, \ldots, j_{s}$ (and also $k_{1}, \ldots, k_{n}$ introduced below). Denote $M=l_{0}+\beta_{j_{1}}-l_{1}+\cdots+\beta_{j_{s}}-l_{s}$. We are at this point considering $M$ as a candidate for $M_{0}$. If some term contributes $x^{M}$ in the expansion of $Q_{0}(y)$, then $M=l_{0}^{\prime}+\beta_{j_{1}^{\prime}}^{\prime}-l_{1}^{\prime}+\cdots+\beta_{j_{s^{\prime}}}-l_{s^{\prime}}^{\prime}$, for a certain term $x^{l_{0}^{\prime}} Y^{\left(l_{1}^{\prime}\right)} \cdots Y^{\left(l_{s}^{\prime}\right)}$ of $Q_{0}$. We therefore must consider representations of the form $M+l=\beta_{j_{1}^{(0)}}+\cdots+\beta_{j_{s}^{(0)}}^{(0)}$ for a finite number (less than $\left.2\left(\operatorname{deg}\left(Q_{0}\right)+1\right)\left(\operatorname{ord}\left(Q_{0}\right)\right)\right)$ 
of integers $l$ such that $|l|<\left(\operatorname{deg}\left(Q_{0}\right)+1\right)\left(\operatorname{ord}\left(Q_{0}\right)\right)$ and $0 \leq s^{(0)} \leq \operatorname{ord}\left(Q_{0}\right) \leq$ $n=\operatorname{ord}(Q)$. Fix some integer $l$ within this range (the subprocedure looks over all such $l$ ). For simplicity of notation assume that $\beta_{j_{1}^{(0)}}, \ldots, \beta_{j_{s}^{(0)}}<0$. Then

$$
\beta_{j_{1}^{(0)}} \geq \frac{M-(\operatorname{deg}(Q)+1)(\operatorname{ord}(Q))}{n+1}=M_{1} .
$$

The subprocedure now considers all $k_{1}$ and for a fixed $k_{1}$, adds the condition to the Tarski algebra formula $\psi_{N}$ that $k_{1}$ is the largest index satisfying the inequality $\beta_{k_{1}} \geq M_{1}$. It then looks over all $j_{1}^{(0)} \leq k_{1}$. Fix some such $j_{1}^{(0)}$ and consider the possible representations of $M+l-\beta_{1}^{(0)}$ in the form $\beta_{j_{2}^{(0)}}+\cdots+\beta_{j_{s^{(0)}}^{(0)}}=M+l-\beta_{j_{1}^{(0)}}$ for $0 \leq s^{(0)} \leq n$, in the same way as above. After $n+1$ steps we will obtain some Tarski algebra formula (for fixed $\left.k_{0}, k_{1}, \ldots, k_{n}\right)$. The formula yields all representations of $M+l$ in the form $M+l=\beta_{j_{1}^{(0)}}+\cdots+\beta_{j_{s^{(0)}}^{(0)}}$ for $0 \leq s^{(0)} \leq n$. Now write down the coefficient of $x^{M}$ in the expansion of $Q_{0}(y)$ and the condition that this coefficient is not zero. This yields some additional Tarski algebra formula which we combine with the existing Tarski algebra formula. Test its satisfiability [TA51, CO75, GR88] and if it is satisfiable, we have $Q_{0}(y) \not \equiv 0\left(\bmod x^{M}\right)$ so $Q_{0}(y)=0$. Our procedure will then output this formula as one of the $\Psi$ in Theorem 4.1. If it is not satisfiable, continue the subprocedure described above for the next $(n+1)$-tuple $\left(k_{0}, \ldots, k_{n}\right)$. If $Q_{0}(y) \not \equiv 0$, then for an appropriate choice of $k_{0}, \ldots, k_{n}$ we will discover that $Q_{0}(y) \neq 0\left(\bmod x^{M}\right)$.

To prove Theorem 4.1 we must show that the above procedure enumerates all the generalized series solutions $y$ of the input equation $P=0$. In expanding $y$ by the Newton polygon process either the active vertex eventually would have ordinate 1 and then $y$ is a solution of the equation $P=0$ ) (by Lemma 2.2), or the active vertex eventually would have ordinate $l>1$ and $\partial^{K} P / \partial Y^{K}=0$ for all $K=\left(k_{1}, \ldots, k_{j}\right)$ with $j<l$ (again by Lemma 2.2). In the second case (see B in the present section) we obtain using [SE56] a new system $Q_{i}=0$, $Q_{i 0} \neq 0$ which has $y$ as a solution and for which $Q_{i} \prec P$. After some steps of this reduction the algorithm comes to a certain system $Q=0, Q_{0} \neq 0$ for which $y$ is a stabilizable solution. This completes the proof of Theorem 4.1.

Corollary. Let $P=0$ be an ordinary differential equation as above. If $P=0$ has a generalized series solution, then it has a solution of the form

$$
y=\sum_{i=0}^{\infty}\left(\bar{\alpha}_{i}+\sqrt{-1} \overline{\bar{\alpha}}_{i}\right) x^{\beta_{i}}
$$

where $\beta_{0}>\beta_{1}>\cdots, \lim _{i \rightarrow \infty} \beta_{i}=-\infty$, and $\bar{\alpha}_{i}, \overline{\bar{\alpha}}_{i}, \beta_{i} \in \widetilde{\mathbb{Q}}$, where $\widetilde{\mathbb{Q}}$ is the real closure of $\mathbb{Q}$. 
Proof. Our assumptions and Theorem 4.1 imply that some Tarski formula $\Psi$ (as in Theorem 4.1) is satisfiable in $\mathbb{R}$. It is well known [TA51] that this implies that $\Psi$ is satisfiable with $\bar{\alpha}_{i}, \overline{\bar{\alpha}}_{i}, \beta_{i}$ in $\widetilde{\mathbb{Q}}$. Theorem 4.1 now implies that the initial segment $\sum_{i=0}^{s}\left(\bar{\alpha}_{i}+\sqrt{-1} \overline{\bar{\alpha}}_{i}\right) x^{\beta_{l}}$ can be extended to a solution $y$ of $P(y)=0$.

Note that the above argument also shows that if $P=0$ has a solution $y=$ $\sum\left(\bar{\alpha}_{i}+\sqrt{-1} \bar{\alpha}_{i}\right) x^{\beta_{i}}$ where $\bar{\alpha}_{i}, \overline{\bar{\alpha}}_{i} \in \mathbb{R}, \beta_{0}>\beta_{1}>\cdots$, and $\beta_{i} \in \mathbb{Z}$, then it has a solution of the same form with $\bar{\alpha}_{i}, \overline{\bar{\alpha}}_{i} \in \widetilde{\mathbb{Q}}$. This yields a result (for a single equation) similar to Theorem 2.7 of [DL84].

\section{DECISION PROBLEMS}

In [DL84] Denef and Lipschitz show that there is an algorithm to decide if a system of polynomial differential equations with coefficients in $\mathbb{Q}[x]$ has a solution in $\mathbb{C}[[x]]$, the ring of formal power series. They also show that, given a system of differential polynomial equations $P_{i}\left(y_{1}, y_{1}^{\prime}, y_{1}^{\prime \prime}, \ldots, y_{m}, y_{m}^{\prime}, \ldots\right)=$ $0, i=1,2,3, \ldots$, with coefficients in $\mathbb{C}[[x]]$ such that for every $n \in$ $\mathbb{N}$ there exists $\overline{y_{1}}, \ldots, \overline{y_{n}}$ in $\mathbb{C}[[x]]$ such that $P_{i}\left(\overline{y_{1}}, \bar{y}_{1}^{\prime}, \ldots, \bar{y}_{m}, \ldots\right)=0$ $\bmod x^{n}, i=1,2,3, \ldots$, then there exist $\overline{\bar{y}}_{1}, \ldots, \overline{\bar{y}}_{m} \in \mathbb{C}[[x]]$ such that $P_{i}\left(\overline{\bar{y}}_{1}, \overline{\bar{y}}_{1}^{\prime}, \ldots \overline{\bar{y}}_{m}, \ldots\right)=0$. In this section we show that this fact is not true in $\Omega$ and also that there is no algorithm to decide if such a system has a solution in $\Omega$. It will be convenient to work with the field

$$
\Omega_{0}=\left\{\sum_{i=0}^{\infty} \alpha_{i} x^{\beta_{i}} \mid \alpha_{i} \in \mathbb{C}, \beta_{i} \in \mathbb{R}, \beta_{0}<\beta_{1}<\cdots, \text { and } \lim _{n \rightarrow \infty} \beta_{i}=\infty\right\}
$$

This field is obviously a differential field with derivation $\frac{d}{d x}$. Using the map that sends $x$ to $x^{-1}$, we see that $\Omega_{0}$ with the derivation $\frac{d}{d x}$ is isomorphic to $\Omega$ with the derivation $D=-x^{2} \frac{d}{d x}$. This observation allows us to transfer results concerning solution of differential equations in $\Omega_{0}$ to similar results about $\Omega$ (and vice versa).

We start by considering the system of differential equations

$$
y^{\prime} x=\beta y, \quad \beta^{\prime}=0, \quad z^{\prime} y x+z^{\prime \prime}=y+x .
$$

We first show that if $\beta=\frac{1}{n}$ for some $n \in \mathbb{N}$, then (4) has a solution in $\Omega_{0}$. If $n=1$, we may let $y=-x$ and $z=0$, so assume $n>1$. We will show that there exist $a_{1}, \ldots, a_{n}$ and $c$ in $\mathbb{C}$ such that $y=c x^{1 / n}$ and $z=a_{1} x^{1 / n}+\cdots+a_{n-1} x^{(n-1) / n}$ are solutions of (4). If we substitute these expressions in the last equation of (4) and compare exponents of $x$, we see 
that

$$
\begin{gathered}
a_{n-1} c\left(\frac{n-1}{n}\right)=1, \\
a_{n-2} c\left(\frac{n-2}{2}\right)+a_{n-1}\left(\frac{n-1}{n}\right)\left(\frac{n-1}{n}-1\right)=0, \\
\vdots \\
a_{1} c+a_{2} \frac{2}{n}\left(\frac{2}{n}-1\right)=0, \\
a_{1} \frac{1}{n}\left(\frac{1}{n}-1\right)=c .
\end{gathered}
$$

These equations determine $c^{n}$ uniquely. Once we select $c$, then $a_{1}, \ldots, a_{n-1}$ are uniquely determined.

Conversely, assume that (4) has a solution in $\Omega_{0}$. We will show that $\beta=\frac{1}{n}$ for some $n \in \mathbb{N}$. The first two equations of (4) imply that $y=c x^{\beta}$ for some $c \in \mathbb{C}$. If $c=0$, then the third equation reduces to $z^{\prime \prime} x^{2}=x$ and this latter equation has no solution in $\Omega_{0}$. Therefore $c \neq 0$ and $\beta \in \mathbb{R}$. Let $\beta \neq 0$, $y=c x^{\beta}$, and $z=\sum_{i=0}^{\infty} \alpha_{i} x^{\beta_{\imath}} \in \Omega_{0}$ be solutions of (4). We shall assume that $\beta \neq \frac{1}{n}$ and derive a contradiction. First we shall show $\beta>0$. If $\beta<0$, comparing lowest powers of $x$ in $z^{\prime} y x+z^{\prime \prime} x=y+x$ yields $c \alpha_{0} \beta_{0} x^{\beta_{0}+\beta}=$ $c x^{\beta}$, which is impossible. Next we shall show that $x^{1-\beta}, x^{1-2 \beta}, x^{1-3 \beta}, \ldots$ must all occur in $z$ with nonzero coefficients. Comparing coefficients of $x^{1}$ in $z^{\prime} y x+z^{\prime \prime} x=y+x$, we have $c \alpha_{i_{1}}(1-\beta)=1$, where $\alpha_{i_{1}}$ is the coefficient of $x^{1-\beta}$ in $z$. Therefore $\alpha_{i_{1}} \neq 0$. Comparing coefficients of $x^{1-n \beta}$, we have $c \alpha_{i_{n}}(1-n \beta)+\alpha_{i_{n-1}}(1-(n-1) \beta)(-(n-1) \beta)=0$ where $\alpha_{i_{n}}$ is the coefficient of $x^{1-n \beta}$ in $z$. Induction shows that $\alpha_{i_{n}} \neq 0$ for all $n$. Since $\lim _{n \rightarrow \infty} 1-n \beta=-\infty$, we see that $z$ cannot lie in $\Omega_{0}^{n}$, a contradiction.

We can now show that the following problem is undecidable: given a system $P_{i}\left(y_{1}, y_{1}^{\prime}, y_{1}^{\prime \prime}, \ldots, y_{m}, y_{m}^{\prime}, \ldots\right)=0, i=1,2, \ldots$, of differential polynomial equations with coefficients in $\mathbb{Q}[x]$, do there exist solutions $y_{1}, \ldots, y_{m} \in \Omega_{0}$ ? Given any diophantine equation $f\left(x_{1}, \ldots, x_{n}\right)=0$ with coefficients in $\mathbb{N}$, consider the system

$$
\begin{gathered}
f\left(\gamma_{1}, \ldots, \gamma_{n}\right)=0, \\
\gamma_{1} \beta_{1}-1=0, \quad \gamma_{2} \beta_{2}-1=0, \ldots, \quad \gamma_{n} \beta_{n}-1=0, \\
\beta_{1}^{\prime}=0, \quad y_{1}^{\prime} x=\beta_{1} y_{1}, \quad z_{1}^{\prime} y_{1} x+z_{1}^{\prime \prime} x=y_{1}+x, \\
\vdots \\
\beta_{n}^{\prime}=0, \quad y_{n}^{\prime} x=\beta_{n} y_{n}, \quad z_{n}^{\prime} y_{n} x+z_{n}^{\prime \prime} x=y_{n}+x .
\end{gathered}
$$

For our discussion above, this system has a solution in $\Omega_{0}$ if and only if $f\left(x_{1}, \ldots, x_{n}\right)=0$ has a solution in $\mathbb{N}-\{0\}$. Since this latter problem is 
known to be undecidable [MA70], our problem is also undecidable. Note that equations (4) have a solution in $\Omega_{0}$ if and only if they have a solution in $\Omega_{0}^{+}$ where $\Omega_{0}^{+}=\left\{\sum_{i=0}^{\infty} \alpha_{i} x^{\beta_{i}} \in \Omega_{0} \mid \beta_{0} \geq 0\right\}$. Therefore the above problem is also undecidable for $\Omega_{0}^{+}$, that is, even if we restrict solutions to have only nonnegative powers of $x$.

The system (4) can also be used to show that a Strong Approximation Theorem (cf. [DL84, p. 224]) does not hold in $\Omega_{0}$. If $P\left(y_{1}, y_{1}^{\prime}, \ldots, y_{m}, y_{m}^{\prime}, \ldots\right)$ is a differential polynomial with coefficients in $\Omega_{0}, \overline{y_{1}}, \ldots, \overline{y_{m}} \in \Omega_{0}$, and $n$ is an integer, we say $P\left(\overline{y_{1}}, \ldots, \overline{y_{m}}, \ldots\right)=0 \bmod x^{n}$ if the lowest power of $x$ occurring in $P\left(\overline{y_{1}}, \ldots, \overline{y_{m}}, \ldots\right)$ has exponent $\geq n$. We say in this case that $\bar{y}_{1}, \ldots, \bar{y}_{n}$ is a solution of $P=0 \bmod x^{n}$. The Strong Approximation Theorem of Denef and Lipschitz states that if, for each positive integer $n$, a system of differential polynomial equations with coefficients in $\mathbb{C}[[x]]$ has a solution $\bmod x^{n}$ in $\mathbb{C}[[x]]$, then the system has a solution in $\mathbb{C}[[x]]$. This is not true for $\Omega_{0}$. The equations $x y^{\prime}-(\alpha+x) y-1=0, \alpha^{\prime}=0$ have solutions

$$
y_{\alpha}=x^{\alpha} e^{x} \int x^{-\alpha-1} e^{-x} d x+c x^{\alpha} e^{x},
$$

where $c$ is an arbitrary constant (cf. [DL84, p. 224]). If $\alpha$ is not in $\mathbb{N}$, then $y \in \Omega_{0}$. If $\alpha$ is in $\mathbb{N}$, then this differential equation does not have a solution in $\Omega_{0}$ (look at the $x^{-1}$ term in $x^{-\alpha-1} e^{-x}$; it yields $\log (x)$ when integrated). On the other hand, given $n$ we can find a large integer $\alpha$ such that the first $\alpha$ terms of $y_{\alpha}$ is a solution $\bmod x^{n}$. Therefore the system

$$
\begin{gathered}
\alpha^{\prime}=0, \quad u^{\prime} x=\alpha u, \quad \alpha \beta=1, \\
z^{\prime} u x+z^{\prime \prime} x^{2}=u+x, \quad x y^{\prime}-(\beta+x) y-1=0
\end{gathered}
$$

has no solution in $\Omega_{0}$ but does have a solution $\bmod x^{n}$ for all positive $n$.

Added in proof. In Lemma 4 of Rat ionalapproximat iont os dut ionsof algebraic differ ent äl equat ions, ,Proc. Amer. Math. Soc. 10 (1959), pp. 238-244, the author, E. R. Kolchin, considers simple zeroes of differential polynomials in valued differential fields (a zero $u$ of $P$ is simple if $\partial P / \partial Y^{(i)}(u) \neq 0$ for some $i$ ). He shows that there is an element $\gamma_{0}$ of the value group such that $|v-u| \geq \gamma_{0}$ for every $v$ in the field which is a zero of $P$ different from $u$. In particular, this implies that generalized series that are simple zeroes of polynomial differential equations are finitely determined. This latter paper uses valuation theoretic techniques but no Newton polygon process.

\section{REFERENCES}

[CO75] G. E. Collins, Quantifier elimination for real closed fields by cylindrical algebraic decomposition, Automata Theory and Formal Languages, 2nd GI Conference, Kaiserslautern ( $\mathrm{H}$. Brakhage, ed.), Lecture Notes in Computer Sci., vol. 33, Springer, 1975, pp. 134-183.

[DE82] J. Della Dora, C. Dicrescenzo, and E. Tournier, An algorithm to obtain formal solutions of a linear homogeneous differential equation at an irregular singular point, EUROCAM '82, Lecture Notes in Computer Sci., vol. 174, Springer-Verlag, 1982, pp. 273-280. 


\section{SOLVING ORDINARY DIFFERENTIAL EQUATIONS}

[DL84] J. Denef and L. Lipshitz, Power series solutions of algebraic differential equations, Math. Ann. 267 (1984), 213-238.

[GR88] D. Yu. Grigor'ev, Complexity of deciding Tarski algebra, J. Symbolic Comput. 5 (1988), 65-108.

[HI76] E. Hille, Ordinary differential equations in the complex domain, Wiley, New York, 1976.

[LA68] D. Laugwitz, Eine nichtarchimedische Erweiterung angeordneter Körper, Math. Nachr. 37 (1968), 225-236.

[LC54] T. Levi-Civita, Sugli infiniti ed infinitesimali actuali quali elementi analytici, Opere Matematiche, vol. 1, Zanichelli, Bologna, 1954, pp. 1-39.

[LR75] A. H. Lightstone and A. Robinson, Nonarchimedian fields and asymptotic expansions, North-Holland, Amsterdam, 1975.

[MA39] S. Mac Lane, The universality of formal power series fields, Bull. Amer. Math. Soc. 45 (1939), 888-890.

[MA70] Yu. Matijasevic, Enumerable sets are diophantine, Dokl. Akad. Nauk SSSR 191 (1970), 279-282; Soviet Math. Dokl. 11 (1970), 354-357.

[OS35] A. Ostrowski, Untersuchungen zur arithmetischen Theorie der Körper, Math. Z. 39 (1935), 269-404.

[RA78] J.-P. Ramis, Dévissage Gevrey, Soc. Math. de France Astérisque 59-60 (1978), 173-204.

[RI66] J. F. Ritt, Differential algebra, Dover, New York, 1966.

[RO73] A. Robinson, Function theory on some nonarchimedian fields, Amer. Math. Monthly 80 (1973), 87-109.

[SE56] A. Seidenberg, An elimination theory for differential algebra, Univ. of California Press, vol. 3, 1956, pp. 31-66.

[TA51] A. Tarski, A decision method for elementary algebra and geometry, Univ. of California Press, 1951.

Leningrad Department of V. A. Steklov Mathematical Institute of the Academy of SCIENCES OF THE USSR, FONTANKA 27, LENINGRAD, 191011 USSR

Department of Mathematics, North Carolina State University, Raleigh, North CarOLINA 27695 